\title{
Chiral effective Lagrangian for doubly charmed baryons up to $\mathcal{O}\left(q^{4}\right)$
}

\author{
Peng-Cheng Qiu $\odot$ and De-Liang Yao®* \\ School of Physics and Electronics, Hunan University, 410082 Changsha, China
}

(Received 26 December 2020; accepted 21 January 2021; published 10 February 2021)

\begin{abstract}
The chiral effective meson-baryon Lagrangian for the description of interactions between the doubly charmed baryons and Goldstone bosons is constructed up to the order of $q^{4}$. The numbers of linearly independent invariant monomials of $\mathcal{O}\left(q^{2}\right), \mathcal{O}\left(q^{3}\right)$, and $\mathcal{O}\left(q^{4}\right)$ are 8,32 , and 218 , in order. The obtained Lagrangian can be used to study the chiral dynamics and relevant phenomenology of the doubly charmed baryons at complete one-loop level in future. For completeness, the nonrelativistic reduction of the Lagrangian is also discussed.
\end{abstract}

DOI: 10.1103/PhysRevD.103.034006

\section{INTRODUCTION}

One of the most crucial tasks in hadron physics is to explore hadrons containing heavy quarks, since they are not only necessary for completing the hadron spectroscopy but also useful for our understanding of the QCD dynamics. In recent years, plenty of heavy-flavored baryons have been observed at experiments, some of which are even exotic beyond the expectation of the conventional quark model, e.g., the $P_{c}$ states as pentaquark candidates reported by LHCb $[1,2]$. Though predicted by the quark model, members of the spectrum of the doubly and triply heavy-flavored baryons are still absent so far, with the only exception being the $\Xi_{c c}^{++}$state. The $\Xi_{c c}^{++}$state is one of the three doubly charmed baryons, showing up in the $\mathbf{2 0}_{M^{-}}$ plet representation of flavor SU(4) group [3] concerning the $u, d, s, c$ quarks. The three baryons are denoted by $\Xi_{c c}^{++}$, $\Xi_{c c}^{+}$, and $\Omega_{c c}^{+}$with quark constituents $[c c u],[c c d]$, and $[c c s]$ in order.

Nevertheless, the establishment of the existence of the doubly charmed baryons is zigzag. It was first reported in 2002 by the SELEX Collaboration [4] that the $\Xi_{c c}^{+}$state was observed with measured mass $3519 \pm 2 \mathrm{MeV}$ [5]. However, this baryon state was not confirmed by any other subsequent experimental groups [6-9]. Moreover, the experimental value is not consistent with theoretical determinations, e.g., by the relativistic quark model [10], effective potential method [11], heavy quark effective theory [12], and lattice QCD [13,14]. Actually, the SELEX result is also questionable according to the analysis

\footnotetext{
*yaodeliang@hnu.edu.cn
}

Published by the American Physical Society under the terms of the Creative Commons Attribution 4.0 International license. Further distribution of this work must maintain attribution to the author(s) and the published article's title, journal citation, and DOI. Funded by SCOAP. based on heavy quark-diquark symmetry; see, e.g., Ref. [15]. Hence, the realistic existence of the doubly charmed baryons, especially the $\Xi_{c c}^{+}$state, becomes very unclear. The issue was addressed in 2017 that the observation of the doubly charged state $\Xi_{c c}^{++}$was announced by the LHCb Collaboration [16], following the theoretical prediction made by Ref. [17]. The reported mass of $\Xi_{c c}^{++}$ is $3621.4 \pm 0.78 \mathrm{MeV}$, which is in good agreement with previous theoretical results within $1 \sigma$ uncertainty $[10,11,14]$. The finding of the $\Xi_{c c}^{++}$state has triggered renewed interest in studying doubly charmed baryons; see, e.g., Refs. [18-22]. Experiments are still ongoing to investigate the properties of the $\Xi_{c c}^{++}$state and also to pursue the other two members, i.e., $\Xi_{c c}^{++}$and $\Omega_{c c}^{+}$, in the family of the doubly charmed baryons. Since the existence of the doubly charmed baryons is now robust, it becomes necessary, on the theoretical side, to investigate them and their excited states using model-independent and systematical methods.

Chiral perturbation theory (ChPT) [23-25] is one such method, which plays a prominent role in the study of the low-energy dynamics of QCD; see, e.g., Refs. [26-29]. It is initially developed for the description of the interactions of the Goldstone bosons stemming from the spontaneous breaking of the $\mathrm{SU}(3)_{L} \times \mathrm{SU}(3)_{R}$ chiral symmetry of QCD [25]. The inclusion of light baryons as degrees of freedom was first done in Ref. [30], and various renormalization versions [31-36] have to be proposed to tackle the power counting problem. In order to study the heavy-flavored hadron spectrum, ChPT can also be extended to describe the interactions between heavy hadrons and Goldstone bosons. Traditionally, ChPT for heavy-flavored hadrons was proposed in Refs. [37-43] by implementing heavyquark symmetry [44] and heavy quark-diquark symmetry $[42,45,46]$ in addition to chiral symmetry, which means a nonrelativistic expansion in terms of the inverse of heavyflavored hadron mass is performed. However, such a 
nonrelativistic expansion distorts the analytic structure of the amplitudes, e.g., the location of the poles of the expanded heavy-flavored hadron propagators are shifted, which could lead to a convergence problem. For instance, the scalar form factor of the nucleon at $t=4 M_{\pi}^{2}$ diverges [34,47]. It is thus more appropriate to utilize relativistic treatment of the matter fields involved in ChPT. For doubly charmed baryons, covariant ChPT analyses can be found in Refs. [48-50], where their masses and electromagnetic form factors were studied at loop level. However, a complete and minimal chiral effective Lagrangian for a full one-loop description of interactions between the doubly charmed baryons and Goldstone bosons is still lacking. In this work, we are going to fill this gap.

This manuscript is organized as follows. In Sec. II, the relevant chiral building blocks are introduced. In Sec. III, the procedure of the construction of the Lagrangian is described in detail. Transformation properties and chiral dimension of the building blocks together with other necessary ingredients such as Clifford algebra elements are shown in Sec. III A. Consequently, invariant monomials are discussed in Sec. III B, while the reduction of the monomials is shown in Sec. III C. Our results of the constructed Lagrangian are listed in Sec. IV, Appendix B, and Appendix C. A short summary is given in Sec. V.

\section{CHIRAL BUILDING BLOCKS OF THE LAGRANGIAN}

QCD is the underlying theory of ChPT and its Lagrangian reads

$$
\mathcal{L}=\mathcal{L}_{\mathrm{QCD}}^{0}+\bar{q} \gamma^{\mu}\left(v_{\mu}+\gamma_{5} a_{\mu}\right) q-\bar{q}\left(s-i \gamma_{5} p\right) q,
$$

where $\mathcal{L}_{\mathrm{QCD}}^{0}$ is the QCD Lagrangian with massless $u, d$, and $s$ quarks. The $\mathcal{L}_{\mathrm{QCD}}^{0}$ exhibits a global $\mathrm{SU}(3)_{L} \times \mathrm{SU}(3)_{R}$ chiral symmetry, which is spontaneously broken to the subgroup $\mathrm{SU}(3)_{V}$ with the emergence of 8 Goldstone bosons according to Goldstone theorem [51]. Here, $v_{\mu}, a_{\mu}, s$, and $p$ are external vector, axial-vector, scalar, and pseudoscalar sources, in order. ${ }^{1}$ The underlying Lagrangian possesses a local SU(3) ${ }_{L} \times \mathrm{SU}(3)_{R}$ chiral symmetry due to the presence of the external fields. Furthermore, the Goldstone bosons acquire little masses from the explicit breaking of the chiral symmetry by setting $s=\operatorname{diag}\left(m_{u}, m_{d}, m_{s}\right)$.

In the chiral effective Lagrangian to be constructed in the following, Goldstone bosons, originating from spontaneously broken chiral symmetry, and the doubly charmed baryons are taken as explicit degrees of freedom. The Goldstone bosons are represented by a matrix $U$, which transforms as

\footnotetext{
${ }^{1}$ Throughout this work, the vector and axial vector currents should be regarded as traceless $3 \times 3$ matrices in the flavor space, i.e., $\left\langle v_{\mu}\right\rangle=\left\langle a_{\mu}\right\rangle=0$.
}

$$
U \rightarrow V_{R} U V_{L}^{+}
$$

under chiral transformation, where $V_{R}$ and $V_{L}$ are independent $\mathrm{SU}(3)$ matrices. The matrix field $U$ is parametrized as

$$
U=\exp (i \sqrt{2} \Phi / F)
$$

with $\Phi$ given by

$$
\Phi=\left(\begin{array}{ccc}
\frac{1}{\sqrt{2}} \pi^{0}+\frac{1}{\sqrt{6}} \eta & \pi^{+} & K^{+} \\
\pi^{-} & -\frac{1}{\sqrt{2}} \pi^{0}+\frac{1}{\sqrt{6}} \eta & K^{0} \\
K^{-} & \bar{K}^{0} & -\frac{2}{\sqrt{6}} \eta
\end{array}\right),
$$

where $F$ is the Goldstone-boson decay constant in the SU(3) chiral limit [52,53]. The doubly charmed baryons with quantum number $J^{P}=\frac{1}{2}^{+}$are collected in the triplet

$$
\psi=\left(\begin{array}{c}
\Xi_{c c}^{++} \\
\Xi_{c c}^{+} \\
\Omega_{c c}^{+}
\end{array}\right),
$$

with $\Xi_{c c}^{++}, \Xi_{c c}^{+}$and $\Omega_{c c}^{+}$denoting the doubly charmed baryons. The triplet transforms as

$$
\psi \rightarrow h\left(V_{R}, V_{L}, U\right) \psi
$$

where the compensator $h\left(V_{R}, V_{L}, U\right)$ is a nonlinear function of the pion field $U, V_{R}$, and $V_{L}$; it is given by

$$
h=\left(\sqrt{V_{R} U V_{L}^{\dagger}}\right)^{\dagger} V_{R} u
$$

with $u=\sqrt{U}$. It is straightforward to derive the chiral transformation property of the corresponding antibaryon fields, which reads

$$
\bar{\psi} \rightarrow \bar{\psi} h^{\dagger}\left(V_{R}, V_{L}, U\right)
$$

For the construction of the chiral effective Lagrangian, it is convenient to use building blocks $X$ which transform in a uniform way [54]:

$$
X \rightarrow h\left(V_{R}, V_{L}, U\right) X h^{\dagger}\left(V_{R}, V_{L}, U\right) .
$$

The building blocks are linear combinations of pion field and external fields. In our case, the following ones are needed:

$$
\begin{aligned}
u_{\mu} & =i\left\{u^{\dagger}\left(\partial_{\mu}-i r_{\mu}\right) u-u\left(\partial_{\mu}-i l_{\mu}\right) u^{\dagger}\right\}, \\
f_{\mu \nu}^{ \pm} & =u F_{\mu \nu}^{L} u^{\dagger} \pm u^{\dagger} F_{\mu \nu}^{R} u, \\
\chi_{ \pm} & =u^{\dagger} \chi u^{\dagger} \pm u \chi^{\dagger} u,
\end{aligned}
$$


where

$$
\begin{aligned}
\chi & =2 B_{0}(s+i p), \quad B_{0}=-\langle 0|\bar{q} q| 0\rangle / 3 F^{2}, \\
F_{\mu \nu}^{R} & =\partial_{\mu} r_{\nu}-\partial_{\nu} r_{\mu}-i\left[r_{\nu}, r_{\mu}\right], \quad r_{\mu}=v_{\mu}+a_{\mu}, \\
F_{\mu \nu}^{L} & =\partial_{\mu} l_{\nu}-\partial_{\nu} l_{\mu}-i\left[l_{\nu}, l_{\mu}\right], \quad l_{\mu}=v_{\mu}-a_{\mu} .
\end{aligned}
$$

Here $\langle 0|\bar{q} q| 0\rangle$ denotes the quark condensate, and $\langle\cdots\rangle$ stands for trace in the flavor space.

The covariant derivative $D^{\mu}$ is defined by

$$
\begin{aligned}
D_{\mu} & =\partial_{\mu}+\Gamma_{\mu}, \\
\Gamma_{\mu} & =\frac{1}{2}\left\{u^{\dagger}\left(\partial_{\mu}-i r_{\mu}\right) u+u\left(\partial_{\mu}-i l_{\mu}\right) u^{\dagger}\right\} .
\end{aligned}
$$

For the covariant derivative acting on any building block $X$, say $\left[D_{\mu}, X\right]$, it can be proved that it transforms as

$$
\left[D_{\mu}, X\right] \rightarrow h\left[D_{\mu}, X\right] h^{\dagger}
$$

by making use of Eq. (7) and the identity $2\left(h \Gamma_{\mu}-\Gamma_{\mu}^{\prime} h\right)=$ $2 \partial_{\mu} h .^{2}$ Nevertheless, for the covariant acting on the baryon field $\psi$, one has

$$
D_{\mu} \psi \rightarrow h\left(D_{\mu} \psi\right), \quad D_{\mu} \bar{\psi} \rightarrow\left(D_{\mu} \bar{\psi}\right) h^{\dagger}
$$

In addition, the following three relations [55]

$$
\begin{aligned}
{\left[D_{\mu}, D_{\nu}\right] X } & =\frac{1}{4}\left[\left[u_{\mu}, u_{\nu}\right], X\right]-\frac{i}{2}\left[f_{\mu \nu}^{+}, X\right], \\
f_{\mu \nu}^{-} & =\left[D_{\mu}, u_{\nu}\right]-\left[D_{\nu}, u_{\mu}\right], \\
h_{\mu \nu} & =\left[D_{\mu}, u_{\nu}\right]+\left[D_{\nu}, u_{\mu}\right],
\end{aligned}
$$

are very useful for the construction of the meson-baryon chiral Lagrangian. The element $h_{\mu \nu}$ on the left-hand side of the third relation can be considered as an extra chiral building block. In consequence, the terms of $\left[D_{\mu}, u_{\nu}\right]$ and $\left[D_{\nu}, u_{\mu}\right]$ can be eliminated by using the last two equalities.

\footnotetext{
${ }^{2}$ Here, $\Gamma_{\mu}^{\prime}$ is given by

$\Gamma_{\mu}^{\prime}=\frac{1}{2}\left(\sqrt{V_{R} U V_{L}^{\dagger}}\right)^{\dagger}\left(\partial_{\mu}-i V_{R} r_{\mu} V_{R}^{\dagger}+V_{R} \partial_{\mu} V_{R}^{\dagger}\right) \sqrt{V_{R} U V_{L}^{\dagger}}$$$
+\frac{1}{2} \sqrt{V_{R} U V_{L}^{\dagger}}\left(\partial_{\mu}-i V_{L} l_{\mu} V_{L}^{\dagger}+V_{L} \partial_{\mu} V_{L}^{\dagger}\right)\left(\sqrt{V_{R} U V_{L}^{\dagger}}\right)^{\dagger} .
$$

TABLE I. Parity $(P)$, charge conjugation $(C)$, Hermitian conjugation (H.c.) transformation properties, and chiral dimension $\left(D_{\chi}\right)$ of the building blocks and the covariant derivative acting on the building blocks. The definitions of $p, c$, and $h$ are shown in Eq. (20), Eq. (24), and Eq. (22).

\begin{tabular}{cccccccc}
\hline \hline & $P$ & $C$ & H.c. & $p$ & $c$ & $h$ & $D_{\chi}$ \\
\hline$u_{\mu}$ & $-u^{\mu}$ & $u_{\mu}^{T}$ & $u_{\mu}$ & 1 & 0 & 0 & 1 \\
$\chi_{+}$ & $\chi_{+}$ & $\chi_{+}^{T}$ & $\chi_{+}$ & 0 & 0 & 0 & 2 \\
$\chi_{-}$ & $-\chi_{-}$ & $\chi_{-}^{T}$ & $-\chi_{-}$ & 1 & 0 & 1 & 2 \\
$f_{+}^{\mu \nu}$ & $f_{+\mu \nu}$ & $-\left(f_{+}^{\mu \nu}\right)^{T}$ & $f_{+}^{\mu \nu}$ & 0 & 1 & 0 & 2 \\
$f_{-}^{\mu \nu}$ & $-f_{-\mu \nu}$ & $\left(f_{-}^{\mu \nu}\right)^{T}$ & $f_{-}^{\mu \nu}$ & 1 & 0 & 0 & 2 \\
$h^{\mu \nu}$ & $-h_{\mu \nu}$ & $\left(h^{\mu \nu}\right)^{T}$ & $h^{\mu \nu}$ & 1 & 0 & 0 & 2 \\
$\vec{D}_{\mu}$ & $\vec{D}^{\mu}$ & $\bar{D}_{\mu}^{T}$ & $\bar{D}_{\mu}$ & 0 & 0 & 0 & 1 \\
\hline \hline
\end{tabular}

\section{CONSTRUCTION OF THE CHIRAL EFFECTIVE LAGRANGIAN}

\section{A. Transformation properties and chiral dimension}

On top of chiral symmetry, the chiral Lagrangian should be invariant under Lorentz transformation, Hermitian conjugation (H.c.), discrete $P$, and $C$ symmetries. ${ }^{3}$ For easy reference, the transformations properties of the building blocks we use are compiled in Table I, which are taken from Refs. [25,54-56]. Moreover, one has to know the power counting of these elements, listed in the last column of Table I, such that invariant monomials of the chiral effective Lagrangian can be organized order by order.

Analogously, the transformation properties and power counting of Clifford algebra elements, the imaginary unit, the metric and Levi-Civita tensors are shown in Table II, which usually appear as ingredients of baryon bilinear $i \bar{\psi} \Gamma \psi$ [54]. The covariant derivative acting on the baryon fields is of zeroth chiral order, since the mass of baryons cannot be deemed as a small quantity in the chiral limit.

\section{B. Invariant monomials}

With the elements specified in Table I and Table II, we are now in the position to construct all the possible invariant terms. The generic form of any invariant monomial, constrained by Lorentz transformation, chiral symmetry, and Hermitian conjugation symmetry, in the effective mesonbaryon chiral Lagrangian can be written as

$$
(i)^{m} \bar{\psi} A \Gamma D^{n} \psi+\text { H.c. }, \quad(m=0,1) .
$$

Here,

\footnotetext{
${ }^{3}$ According to the $C P T$ theorem, any Lorentz invariant term one can write down in the Lagrangian is $C P T$ invariant. Hence, the time reversal invariance is automatically embedded, once Lorentz covariance, Hermitian conjugation, spatial inversion, and charge conjugation symmetry are implemented in constructing the chiral local Lagrangian.
} 
TABLE II. Parity $(P)$, charge conjugation $(C)$, Hermitian conjugation (H.c.) transformation properties, and chiral dimension $\left(D_{\chi}\right)$ of the Clifford algebra elements, the metric and LeviCivita tensors together with the imaginary unit and the covariant derivative acting on the baryon fields. The definitions of $p, c$ and $h$ are shown in Eq. (20), Eq. (24), and Eq. (22).

\begin{tabular}{llllc}
\hline \hline & $p$ & $c$ & $h$ & $D_{\chi}$ \\
\hline$i$ & 0 & 0 & 1 & 0 \\
1 & 0 & 0 & 0 & 0 \\
$\gamma_{5}$ & 1 & 0 & 1 & 1 \\
$\gamma_{\mu}$ & 0 & 1 & 0 & 0 \\
$\gamma_{5} \gamma_{\mu}$ & 1 & 0 & 0 & 0 \\
$\sigma_{\mu \nu}$ & 0 & 1 & 0 & 0 \\
$g_{\mu \nu}$ & 0 & 0 & 0 & 0 \\
$\epsilon_{\mu \nu \rho \tau}$ & 1 & 0 & 0 & 0 \\
$\vec{D}_{\mu} \psi$ & 0 & 1 & 1 & 0 \\
\hline \hline
\end{tabular}

(i) $A$ is a product of building blocks and their covariant derivatives. Since the matrix fields do not commute with each other, one should consider all possible permutations. In addition, it is more preferable to express all the products in terms of combinations of (anti)commutators. For instance, $u_{\mu} u_{\nu}$ can be written as $u_{\mu} u_{\nu}=\left(\left\{u_{\mu}, u_{\nu}\right\}+\left[u_{\mu}, u_{\nu}\right]\right) / 2$.

(ii) $\Gamma$ is a product of elements of the Clifford algebra basis and/or the metric tensors, the Levi-Civita tensors. More discussions on $\Gamma$ are shown in Appendix A.

(iii) $D^{n} \equiv\left[D^{\mu_{1}} D^{\mu_{2}} \cdots D^{\mu_{n}}+\right.$ all the other permutations $]$ is a product of $n \geq 0$ covariant derivatives acting on $\psi$ in a totally symmetrized way. It is worth pointing out that the commutators of the covariant derivatives can be translated to the basic building blocks with the help of Eq. (16).

Note that, in Eq. (19), the Lorentz indices are suppressed for brevity. However, one needs to keep in mind that all the Lorentz indices of $A$ must be properly contracted with those coming from $\Gamma$ and $D^{n}$ to guarantee that all the terms are Lorentz scalars.

Let us proceed with the transformation property of Eq. (19) under parity, which reads

$$
\begin{aligned}
& \left\{(i)^{m} \bar{\psi} A \Gamma D^{n} \psi+\text { H.c. }\right\}^{P} \\
& \quad=(-1)^{p_{A}+p_{\Gamma}+p_{D}}\left\{(i)^{m} \bar{\psi} A \Gamma D^{n} \psi+\text { H.c. }\right\},
\end{aligned}
$$

where $p_{A}$ can be determined from Table I, while $p_{\Gamma}$ and $p_{D}$ from Table II. It can be concluded from Eq. (20) that the monomial of Eq. (19) survives in the chiral effective chiral Lagrangian only if

$$
(-1)^{p_{A}+p_{\Gamma}+p_{D}}=1
$$

Although Eq. (19) is obviously invariant under Hermitic conjugation, it is still worthwhile to demonstrate the corresponding transformation property explicitly. Equation (19) can be rewritten as

$$
\begin{aligned}
& (i)^{m} \bar{\psi} A \Gamma D^{n} \psi+(-1)^{h_{A}+h_{\Gamma}+m}(i)^{m} \bar{\psi} \overleftarrow{D}^{n} A \Gamma \psi \\
& \quad=(i)^{m} \bar{\psi} A \Gamma D^{n} \psi+(-1)^{h_{A}+h_{\Gamma}+h_{D}+m}(i)^{m} \bar{\psi} A \Gamma D^{n} \psi+\text { h.o., }
\end{aligned}
$$

where the values of $h_{A}, h_{\Gamma}$, and $h_{D}$ are calculated with the help of Table I and Table II. Note that the value of $h_{A}$ includes an additional factor of 1 for each commutator. The terms on the right-hand side of Eq. (22) is obtained by making use of integration by parts and the Leibniz rule together with the elimination of total derivatives. The last term h.o. denotes the sum of higher order pieces with covariant derivatives acting on the building blocks shown Table I, and hence actually can be thrown away from the Lagrangian of a given chiral order under construction. Finally, Eq. (19) can occur in the Lagrangian only if

$$
(-1)^{h_{A}+h_{\Gamma}+h_{D}+m}=1 .
$$

Lastly, we check the invariance of Eq. (22), equivalent to Eq. (19), under charge conjugation,

$$
\begin{aligned}
& \left\{2(i)^{m} \bar{\psi} A \Gamma D^{n} \psi+\text { h.o. }\right\}^{C} \\
& \quad=(-1)^{c_{A}+c_{\Gamma}+c_{D}} 2(i)^{m} \bar{\psi} A \Gamma D^{n} \psi+\text { h.o. }
\end{aligned}
$$

where $c_{A}$, which contains an extra factor of 1 for each commutator, is determined from Table I. Meanwhile, the values of $c_{\Gamma}+c_{D}$ can be obtained from Table II. Likewise, for a given chiral order, Eq. (19) remains in the Lagrangian only if

$$
(-1)^{c_{A}+c_{\Gamma}+c_{D}}=1 .
$$

\section{Reduction of the monomials}

A list of invariant monomials, some of them might be linearly dependent, can be obtained according to the procedure discussed above. In this section, we will utilize several linear identities to remove these dependent monomials. The first relation stems from the property of matrix trace, namely,

$$
\langle a b c\rangle=\langle b c a\rangle=\langle c a b\rangle .
$$

The second relation is the Schouten identity

$$
\epsilon^{\mu \nu \lambda \tau} a^{\rho}+\epsilon^{\nu \lambda \tau \rho} a^{\mu}+\epsilon^{\lambda \tau \rho \mu} a^{\nu}+\epsilon^{\tau \rho \mu \nu} a^{\lambda}+\epsilon^{\rho \mu \nu \lambda} a^{\tau}=0 .
$$

The third relation is obtained by making use of the Bianchi identity, Eq. (16) and Eq. (17), 


$$
\begin{aligned}
& {\left[D_{\mu}, f_{\nu \lambda}^{ \pm}\right]+\left[D_{\nu}, f_{\lambda \mu}^{ \pm}\right]+\left[D_{\lambda}, f_{\mu \nu}^{ \pm}\right]} \\
& \quad=\frac{i}{2}\left[u_{\mu}, f_{\nu \lambda}^{\mp}\right]+\frac{i}{2}\left[u_{\nu}, f_{\lambda \mu}^{\mp}\right]+\frac{i}{2}\left[u_{\lambda}, f_{\mu \nu}^{\mp}\right] .
\end{aligned}
$$

It can be used to eliminate certain monomials containing $\left[D_{\mu}, f_{\nu \lambda}^{ \pm}\right]$or $\left[D_{\nu}, f_{\lambda \mu}^{ \pm}\right],\left[D_{\lambda}, f_{\mu \nu}^{ \pm}\right]$, as done in Ref. [57].

The fourth relation is the so-called Cayley-Hamilton relation [56]. For any $3 \times 3$ arbitrary matrices $a, b$, and $c$, the Cayley-Hamilton relation indicates that

$$
\begin{aligned}
& a b c+b c a+c a b+a c b+c b a+b a c-a b\langle c\rangle-b c\langle a\rangle \\
& \quad-c a\langle b\rangle-a c\langle b\rangle-c b\langle a\rangle-b a\langle c\rangle-a\langle b c\rangle-b\langle c a\rangle \\
& -c\langle a b\rangle-\langle a b c\rangle-\langle a c b\rangle+a\langle b\rangle\langle c\rangle+b\langle c\rangle\langle a\rangle \\
& +c\langle a\rangle\langle b\rangle+\langle a\rangle\langle b c\rangle+\langle b\rangle\langle c a\rangle \\
& +\langle c\rangle\langle a b\rangle-\langle a\rangle\langle b\rangle\langle c\rangle=0,
\end{aligned}
$$

which is usually adopted to replace the terms with two or more traces by those with one trace or without trace.

On the other hand, it is also possible to use equations of motion (EOM) to remove some redundant terms. First of all, the lowest order EOM of the pseudoscalar meson reads

$$
D_{\mu} u^{\mu}=\frac{i}{2} \tilde{\chi}_{-}
$$

where

$$
\tilde{\chi}_{-}=\chi_{-}-\frac{1}{3}\left\langle\chi_{-}\right\rangle
$$

With Eq. (16), Eq. (17), and the EOM of Eq. (30), one can prove that

$$
D^{2} u_{\mu}=\frac{1}{4}\left[\left[u_{\nu}, u_{\mu}\right], u^{\nu}\right]-\frac{i}{2}\left[f_{\nu \mu}^{+}, u^{\nu}\right]+D^{\nu} f_{\nu \mu}^{-}+\frac{i}{2} D_{\mu} \tilde{\chi}_{-} .
$$

Therefore, $D_{\mu} u^{\mu}$ and $D^{2} u_{\mu}$ cannot be regarded as independent structures $[25,52]$.

The lowest order EOMs of the baryon fields, obtainable from the Lagrangian in Eq. (47) to be shown in the next section, are

$$
\begin{aligned}
& \left(i \not D-m+\frac{g_{A}}{2} \not l \gamma_{5}\right) \psi=0, \\
& \bar{\psi}\left(i \not \grave{D}+m-\frac{g_{A}}{2} \not l \gamma_{5}\right)=0,
\end{aligned}
$$

where $(i \not D-m)$ is counted as $O(q)$. Here $m$ and $g_{A}$ are the mass and the axial coupling of the doubly charmed baryons in the $\mathrm{SU}(3)$ chiral limit, respectively. Based on the above two equations, one can obtain a few linear relations to eliminate many unnecessary terms. The linear relations we use read [55,57]:

$$
\begin{aligned}
& \bar{\psi} A^{\mu} i D_{\mu} \psi+\text { H.c. } \doteq 2 m \bar{\psi} \gamma_{\mu} A^{\mu} \psi, \\
& \bar{\psi} \gamma_{\mu}\left[i D^{\mu}, A\right] \psi \doteq \frac{g_{A}}{2} \bar{\psi} \gamma^{\mu} \gamma_{5}\left[A, u_{\mu}\right] \psi, \\
& \bar{\psi} \gamma_{5} \gamma_{\mu}\left[i D^{\mu}, A\right] \psi \doteq-2 m \bar{\psi} \gamma_{5} A \psi-\frac{g_{A}}{2} \bar{\psi} \gamma^{\mu}\left[A, u_{\mu}\right] \psi, \\
& \bar{\psi} \gamma_{5} \gamma_{\lambda} A^{\mu \lambda} i D_{\mu} \psi+\text { H.c. } \doteq 2 i m \bar{\psi} \gamma_{5} \sigma_{\mu \lambda} A^{\mu \lambda} \psi \\
&+\left(\bar{\psi} \gamma_{5} \gamma_{\mu} A^{\mu \lambda} i D_{\lambda} \psi+\text { H.c. }\right), \\
& \bar{\psi} \gamma_{5} \gamma_{\lambda} A^{\mu \lambda} i D_{\mu} \psi+\text { H.c. } \doteq m \bar{\psi} \sigma^{\nu \rho} \epsilon_{\nu \rho \mu \lambda} A^{\mu \lambda} \psi \\
&+\left(\bar{\psi} \gamma_{5} \gamma_{\mu} A^{\mu \lambda} i D_{\lambda} \psi+\text { H.c. }\right), \\
& \bar{\psi} \sigma_{\alpha \beta} A^{\alpha \beta \mu} i D_{\mu} \psi+\text { H.c. } \doteq-2 m \bar{\psi} \epsilon_{\alpha \beta \mu \nu} \gamma_{5} \gamma^{\nu} A^{\alpha \beta \mu} \psi \\
&-\left(\bar{\psi} \sigma_{\beta \mu} A^{\alpha \beta \mu} i D_{\alpha} \psi+\text { H.c. }\right) \\
&+\left(\bar{\psi} \sigma_{\alpha \mu} A^{\alpha \beta \mu} i D_{\beta} \psi+\text { H.c. }\right),
\end{aligned}
$$

where the symbol $\doteq$ means the two objects on the left- and right-hand sides are equal up to some negligible higher order pieces.

\section{Nonrelativistic projection}

For completeness, we further consider the doubly charmed baryons as heavy static sources in the nonrelativistic limit and perform the so-called heavy baryon (HB) projection [31] of the relativistic Lagrangian. Here, we present a brief introduction to the nonrelativistic approach (for more detailed discussions, see, e.g., Refs. [26,31,32]).

The four-momentum of the doubly charmed baryon can be split as

$$
p_{\mu}=m v_{\mu}+l_{\mu}
$$

where $v_{\mu}$ denotes the four-velocity satisfying $v^{2}=1$ and $l_{\mu}$ is the small off-shell momentum with $v \cdot l \ll m$. The doubly charmed baryon field $\psi$ can be decomposed into large component $H$ and small component $h$ via

$$
\psi=e^{-i m v \cdot x}(H+h),
$$

with

$$
\not H=H, \quad \not h=-h .
$$

In terms of $H$ and $h$, the meson-baryon chiral Lagrangian can be recast as

$$
\mathcal{L}=\bar{H} \mathcal{A} H+\bar{h} \mathcal{B} H+\bar{H} \gamma_{0} \mathcal{B}^{\dagger} \gamma_{0} h-\bar{h} \mathcal{C} h
$$


where the operators $\mathcal{A}, \mathcal{B}$, and $\mathcal{C}$ may be expanded as a series of the low energy momentum $q$. In this approach, it is more advantageous to make use of the velocity $v_{\mu}$ and the spin-operator $S_{\mu}=\frac{i}{2} \gamma_{5} \sigma_{\mu \nu} v^{\nu}$ to express every baryon bilinear $\bar{\psi} \Gamma \psi$.

After eliminating the small component $h$, the nonrelativistic Lagrangian reads

$$
\mathcal{L}=\bar{H}\left\{\mathcal{A}+\left(\gamma_{0} \mathcal{B}^{\dagger} \gamma_{0}\right) \mathcal{C}^{-1} \mathcal{B}\right\} H
$$

where

$$
\begin{aligned}
\mathcal{C}^{-1}= & \frac{1}{2 m}-\frac{i(v \cdot D)+g_{A} S \cdot u}{(2 m)^{2}}+\frac{\left(i v \cdot D+g_{A} S \cdot u\right)^{2}}{(2 m)^{3}} \\
& -\frac{\mathcal{C}^{(2)}}{(2 m)^{2}}+\cdots .
\end{aligned}
$$

\section{THE CHIRAL EFFECTIVE MESON-BARYON LAGRANGIAN}

Using the method described above, we have constructed the minimal and complete chiral effective meson-baryon Lagrangians up to $O\left(q^{4}\right)$ both in the relativistic and the nonrelativistic forms.

\section{A. The Lagrangian at $\boldsymbol{O}(\boldsymbol{q})$}

The lowest order of relativistic chiral effective Lagrangian reads

$$
\mathcal{L}_{M \psi}^{(1)}=\bar{\psi}(i \not D-m) \psi+\frac{g_{A}}{2} \bar{\psi} \psi / \gamma_{5} \psi
$$

where $m$ is the mass of baryon and $g_{A}$ is the axial-vector coupling constant in the $\mathrm{SU}(3)$ chiral limit. The operator $(i \not D-m)$ is counted as $O(q)$ in the chiral expansion, as discussed in Ref. [54]. In principle, the $g_{A}$ coupling is an unknown parameter which needs to be determined by experimental data. In Ref. [48], it was estimated to be $\left|g_{A}\right|=0.2$. The above leading order Lagrangian has been used to explore the possible exotic states in the spectrum of doubly charmed baryons [58].

The corresponding non-relativistic Lagrangian can be expressed as

$$
\hat{\mathcal{L}}_{M \psi}^{(1)}=\bar{H}\left(i v \cdot D+g_{A} S \cdot u\right) H
$$

In the nonrelativistic Lagrangian of leading order, the doubly charmed baryon mass term disappears and the Dirac matrices have been substituted by $v_{\mu}$ and $S_{\mu}$.
TABLE III. Terms in the relativistic and nonrelativistic Lagrangian of $O\left(q^{2}\right)$.

\begin{tabular}{lcc}
\hline \hline$i$ & $O_{i}^{(2)}$ & $\hat{O}_{i}^{(2)}$ \\
\hline 1 & $\left\langle\chi_{+}\right\rangle$ & $\left\langle\chi_{+}\right\rangle$ \\
2 & $\tilde{\chi}_{+}$ & $\tilde{\chi}_{+}$ \\
3 & $u^{2}$ & $u^{2}$ \\
4 & $\left\langle u^{2}\right\rangle$ & $\left\langle u^{2}\right\rangle$ \\
5 & $\left\{u^{\mu}, u^{\nu}\right\} D_{\mu \nu}+$ H.c. & $-8 m^{2}(v \cdot u)^{2}$ \\
6 & $\left\langle u^{\mu} u^{\nu}\right\rangle D_{\mu \nu}+$ H.c. & $-4 m^{2}\left\langle(v \cdot u)^{2}\right\rangle$ \\
7 & $i\left[u^{\mu}, u^{\nu}\right] \sigma_{\mu \nu}$ & $2\left[S_{\mu}, S_{\nu}\right]\left[u^{\mu}, u^{\nu}\right]$ \\
8 & $f_{+}^{\mu \nu} \sigma_{\mu \nu}$ & $-2 i\left[S_{\mu}, S_{\nu}\right] f_{+}^{\mu \nu}$ \\
\hline \hline
\end{tabular}

\section{B. The Lagrangian at $O\left(q^{2}\right)$}

The $O\left(q^{2}\right)$ meson-baryon Lagrangian can be written as

$$
\mathcal{L}_{M \psi}^{(2)}=\sum_{i=1}^{8} b_{i} \bar{\psi} O_{i}^{(2)} \psi
$$

where $b_{i}$ 's are unknown low-energy constants (LECs). It was pointed out by Ref. [59] that some of the $O\left(q^{2}\right)$ LECs can be related to those in the charmed meson Lagrangian [60] by imposing heavy antiquark-diquark symmetry [61]. The monomials $O_{i}^{(2)}$ are given in the 2nd column of Table III. ${ }^{4}$

In nonrelativistic form, the chiral Lagrangian of $O\left(q^{2}\right)$ reads

$$
\hat{\mathcal{L}}_{M \psi}^{(2)}=\bar{H}\left\{\mathcal{A}^{(2)}+\gamma_{0} \mathcal{B}^{(1) \dagger} \gamma_{0} \mathcal{C}^{(0)-1} \mathcal{B}^{(1)}\right\} H,
$$

where

$$
\mathcal{A}^{(2)}=\sum_{i=1}^{8} b_{i} \hat{O}_{i}^{(2)}
$$

The non-relativistic operators $\hat{O}_{i}^{(2)}$ corresponding to the relativistic monomials $O_{i}^{(2)}$ are listed in the 3rd column of Table III. The second term in the bracket incorporates the $1 / m$ corrections, which are shown in Appendix B. Explicit expressions for $\mathcal{B}^{(i)}$ and $\mathcal{C}^{(i)}$ are collected in Appendix B as well. Based on the Lagrangian we obtained here, we have checked that it is straightforward to reproduce the pion-nucleon Lagrangian in Ref. [57] by using the Cayley-Hamilton relation for $2 \times 2$ matrices.

\footnotetext{
${ }^{4}$ There is one more term in the $O\left(q^{2}\right)$ Lagrangian given by Ref. [62]. However, terms with $\left\langle f_{\mu \nu}^{+}\right\rangle$do not show up in our case, due to the fact that the external vector and axial vector currents are set to be traceless.
} 


\section{The Lagrangian at $O\left(q^{3}\right)$}

The chiral meson-baryon Lagrangian at $O\left(q^{3}\right)$ takes the form

$$
\mathcal{L}_{M \psi}^{(3)}=\sum_{i=1}^{32} c_{i} \bar{\psi} O_{i}^{(3)} \psi
$$

where $c_{i}$ are $O\left(q^{3}\right)$ LECs and the operators $O_{i=1, \cdots 32}^{(3)}$ are listed in the 2nd column of Table IV.

Also, the $O\left(q^{3}\right)$ nonrelativistic Lagrangian can be written as

$$
\begin{aligned}
\hat{\mathcal{L}}_{M \psi}^{(3)}= & \bar{H}\left\{\mathcal{A}^{(3)}+\gamma_{0} \mathcal{B}^{(1) \dagger} \gamma_{0} \mathcal{C}^{(0)-1} \mathcal{B}^{(2)}+\gamma_{0} \mathcal{B}^{(1) \dagger} \gamma_{0} \mathcal{C}^{(1)-1} \mathcal{B}^{(1)}\right. \\
& \left.+\gamma_{0} \mathcal{B}^{(2) \dagger} \gamma_{0} \mathcal{C}^{(0)-1} \mathcal{B}^{(1)}\right\} H,
\end{aligned}
$$

with

$$
\mathcal{A}^{(3)}=\sum_{i=1}^{32} c_{i} \hat{O}_{i}^{(3)}
$$

\begin{tabular}{|c|c|c|}
\hline$i$ & $O_{i}^{(3)}$ & $\hat{O}_{i}^{(3)}$ \\
\hline 1 & $\left\{u_{\mu},\left\{u^{\mu}, u^{\nu}\right\}\right\} \gamma_{5} \gamma_{\nu}$ & $-2\left\{u_{\mu},\left\{u^{\mu}, S \cdot u\right\}\right\}$ \\
\hline 2 & {$\left[u_{\mu},\left[u^{\mu}, u^{\nu}\right]\right] \gamma_{5} \gamma_{\nu}$} & $-2\left[u_{\mu},\left[u^{\mu}, S \cdot u\right]\right]$ \\
\hline 3 & $u^{\nu} \gamma_{5} \gamma_{\nu}\left\langle u^{2}\right\rangle$ & $-2 S \cdot u\left\langle u^{2}\right\rangle$ \\
\hline 4 & $u_{\mu} \gamma_{5} \gamma_{\nu}\left\langle u^{\mu} u^{\nu}\right\rangle$ & $-2 u_{\mu}\left\langle u^{\mu} S \cdot u\right\rangle$ \\
\hline 5 & $\left\{u^{\mu},\left\{u^{\nu}, u^{\rho}\right\}\right\} \gamma_{5} \gamma_{\mu} D_{\nu \rho}+$ H.c. & $\left.16 m^{2}\left\{S \cdot u,(v \cdot u)^{2}\right\}\right\}$ \\
\hline 6 & $u^{\mu} \gamma_{5} \gamma_{\mu}\left\langle u^{\nu} u^{\rho}\right\rangle D_{\nu \rho}+$ H.c. & $8 m^{2} S \cdot u\left\langle(v \cdot u)^{2}\right\rangle$ \\
\hline 7 & $i \epsilon_{\mu \nu \rho \tau}\left\{\left[u^{\mu}, u^{\nu}\right], u^{\rho}\right\} \gamma^{\tau}$ & $i \epsilon_{\mu \nu \rho \tau}\left\{\left[u^{\mu}, u^{\nu}\right], u^{\rho}\right\} v^{\tau}$ \\
\hline 8 & $i \epsilon_{\mu \nu \rho \tau} \gamma^{\tau}\left\langle\left[u^{\mu}, u^{\nu}\right] u^{\rho}\right\rangle$ & $i \epsilon_{\mu \nu \rho \tau} v^{\tau}\left\langle\left[u^{\mu}, u^{\nu}\right] u^{\rho}\right\rangle$ \\
\hline 9 & $i \epsilon_{\mu \nu \lambda \tau}\left\{u^{\mu},\left\{u^{\nu}, u^{\rho}\right\}\right\} \sigma^{\lambda \tau} D_{\rho}+$ H.c. & $-4 i m \epsilon_{\mu \nu \lambda \tau}\left\{u^{\mu},\left\{u^{\nu}, v \cdot u\right\}\right\}\left[S^{\lambda}, S^{\tau}\right]$ \\
\hline 10 & $i \epsilon_{\mu \nu \lambda \tau} u^{\mu} \sigma^{\lambda \tau}\left\langle u^{\nu} u^{\rho}\right\rangle D_{\rho}+$ H.c. & $-4 i m \epsilon_{\mu \nu \lambda \tau} u^{\mu}\left\langle u^{\nu} v \cdot u\right\rangle\left[S^{\lambda}, S^{\tau}\right]$ \\
\hline 11 & $i\left[u_{\mu}, h^{\mu \nu}\right] \gamma_{\nu}$ & $i\left[u_{\mu}, h^{\mu \nu}\right] v_{\nu}$ \\
\hline 12 & $i\left[u^{\mu}, h^{\nu \rho}\right] \gamma_{\mu} D_{\nu \rho}+$ H.c. & $-4 \operatorname{im}^{2}\left[v \cdot u, h^{\nu \rho}\right] v_{\nu} v_{\rho}$ \\
\hline 13 & $i\left\{u^{\mu}, h^{\nu \rho}\right\} \sigma_{\mu \nu} D_{\rho}+$ H.c. & $-4 i m\left\{u^{\mu}, h^{\nu \rho}\right\}\left[S_{\mu}, S_{\nu}\right] v_{\rho}$ \\
\hline 14 & $i \sigma_{\mu \nu}\left\langle u^{\mu} h^{\nu \rho}\right\rangle D_{\rho}+$ H.c. & $-4 i m\left[S_{\mu}, S_{\nu}\right]\left\langle u^{\mu} h^{\nu \rho}\right\rangle v_{\rho}$ \\
\hline 15 & $\left\{u^{\mu}, \tilde{\chi}_{+}\right\} \gamma_{5} \gamma_{\mu}$ & $-2\left\{S \cdot u, \tilde{\chi}_{+}\right\}$ \\
\hline 16 & $u^{\mu} \gamma_{5} \gamma_{\mu}\left\langle\chi_{+}\right\rangle$ & $-2 S \cdot u\left\langle\chi_{+}\right\rangle$ \\
\hline 17 & $\gamma_{5} \gamma_{\mu}\left\langle u^{\mu} \tilde{\chi}_{+}\right\rangle$ & $-2\left\langle S \cdot u \tilde{\chi}_{+}\right\rangle$ \\
\hline 18 & $i \gamma_{5} \gamma_{\mu}\left[D^{\mu}, \tilde{\chi}_{-}\right]$ & $-2 i\left[S \cdot D, \tilde{\chi}_{-}\right]$ \\
\hline 19 & $i \gamma_{5} \gamma_{\mu}\left\langle\left[D^{\mu}, \chi_{-}\right]\right\rangle$ & $-2 i\left\langle\left[S \cdot D, \chi_{-}\right]\right\rangle$ \\
\hline 20 & {$\left[\tilde{\chi}_{-}, u^{\mu}\right] \gamma_{\mu}$} & {$\left[\tilde{\chi}_{-}, v \cdot u\right]$} \\
\hline 21 & $i\left[u_{\mu}, f_{+}^{\mu \nu}\right] \gamma_{5} \gamma_{\nu}$ & $-2 i\left[u_{\mu}, f_{+}^{\mu \nu}\right] S_{\nu}$ \\
\hline 22 & $\epsilon_{\mu \nu \rho \tau}\left\{u^{\mu}, f_{+}^{\nu \rho}\right\} \gamma^{\tau}$ & $\epsilon_{\mu \nu \rho \tau}\left\{u^{\mu}, f_{+}^{\nu \rho}\right\} v^{\tau}$ \\
\hline 23 & $\epsilon_{\mu \nu \rho \tau} \gamma^{\tau}\left\langle u^{\mu} f_{+}^{\nu \rho}\right\rangle$ & $\epsilon_{\mu \nu \rho \tau} v^{\tau}\left\langle u^{\mu} f_{+}^{\nu \rho}\right\rangle$ \\
\hline 24 & $\epsilon_{\nu \rho \lambda \tau}\left[u^{\mu}, f_{+}^{\nu \rho}\right] \sigma^{\lambda \tau} D_{\mu}+$ H.c. & $-4 m \epsilon_{\nu \rho \lambda \tau}\left[v \cdot u, f_{+}^{\nu \rho}\right]\left[S^{\lambda}, S^{\tau}\right]$ \\
\hline 25 & $i\left[D_{\mu}, f_{+}^{\mu \nu}\right] D_{\nu}+$ H.c. & $2 m\left[D_{\mu}, f_{+}^{\mu \nu}\right] v_{\nu}$ \\
\hline 26 & $i\left[u_{\mu}, f_{-}^{\mu \nu}\right] \gamma_{\nu}$ & $i\left[u_{\mu}, f_{-}^{\mu \nu}\right] v_{\nu}$ \\
\hline 27 & $\epsilon_{\mu \nu \rho \tau}\left\{u^{\mu}, f_{-}^{\nu \rho}\right\} \gamma_{5} \gamma^{\tau}$ & $-2 \epsilon_{\mu \nu \rho \tau}\left\{u^{\mu}, f^{\nu \rho}\right\} S^{\tau}$ \\
\hline 28 & $\epsilon_{\mu \nu \rho \tau} \gamma_{5} \gamma^{\tau}\left\langle u^{\mu} f_{-}^{\nu \rho}\right\rangle$ & $-2 \epsilon_{\mu \nu \rho \tau} S^{\tau}\left\langle u^{\mu} f_{-}^{\nu \rho}\right\rangle$ \\
\hline 29 & $i\left\{u^{\mu}, f_{-}^{\nu \rho}\right\} \sigma_{\mu \nu} D_{\rho}+$ H.c. & $-4 i m\left\{u^{\mu}, f_{-}^{\nu \rho}\right\}\left[S_{\mu}, S_{\nu}\right] v_{\rho}$ \\
\hline 30 & $i \sigma_{\mu \nu}\left\langle u^{\mu} f_{-}^{\nu \rho}\right\rangle D_{\rho}+$ H.c. & $-4 i m\left[S_{\mu}, S_{\nu}\right] v_{\rho}\left\langle u^{\mu} f_{-}^{\nu \rho}\right\rangle$ \\
\hline 31 & {$\left[D_{\mu}, f_{-}^{\mu \nu}\right] \gamma_{5} \gamma_{\nu}$} & $-2 S_{\nu}\left[D_{\mu}, f_{-}^{\mu \nu}\right]$ \\
\hline 32 & {$\left[D^{\lambda}, f_{-}^{\mu \nu}\right] \gamma_{5} \gamma_{\mu} D_{\nu \lambda}+$ H.c. } & $8 m^{2} S_{\mu}\left[v \cdot D, f_{-}^{\mu \nu}\right] v_{\nu}$ \\
\hline
\end{tabular}

where the monomials $\hat{O}_{i}^{(3)}$ are collected in the 3rd column of Table IV. The $1 / \mathrm{m}$ corrections are given in Appendix B.

TABLE IV. Terms in the $O\left(q^{3}\right)$ relativistic and nonrelativistic Lagrangians. 


\section{The Lagrangian at $O\left(q^{4}\right)$}

The chiral effective meson-baryon Lagrangian at $O\left(q^{4}\right)$ reads

$$
\mathcal{L}_{M \psi}^{(4)}=\sum_{i=1}^{218} d_{i} \bar{\psi} O_{i}^{(4)} \psi
$$

where $d_{i}$ are LECs and the terms of $O\left(q^{4}\right)$ are tabulated in the 2nd column of Table V. The last three terms are contact terms (See Refs. $[56,63]$ for analogous operators). It should be noted that they are combinations depending only on external fields and therefore are not directly accessible experimentally. Furthermore, as pointed out by Ref. [56], it is more convenient to express the contact terms in terms of the LR basis, i.e., $F_{R / L}^{\mu \nu}$, and the $\chi$ element, rather than those chiral building blocks in Table I.

The $O\left(q^{4}\right)$ meson-baryon nonrelativistic Lagrangian which contains the $1 / m$ corrections takes the form

$$
\begin{aligned}
\hat{\mathcal{L}}_{M \psi}^{(4)}= & \bar{H}\left\{\mathcal{A}^{(4)}+\gamma_{0} \mathcal{B}^{(1) \dagger} \gamma_{0} \mathcal{C}^{(0)-1} \mathcal{B}^{(3)}+\gamma_{0} \mathcal{B}^{(1) \dagger} \gamma_{0} \mathcal{C}^{(1)-1} \mathcal{B}^{(2)}\right. \\
& +\gamma_{0} \mathcal{B}^{(1) \dagger} \gamma_{0} \mathcal{C}^{(2)-1} \mathcal{B}^{(1)}+\gamma_{0} \mathcal{B}^{(2) \dagger} \gamma_{0} \mathcal{C}^{(0)-1} \mathcal{B}^{(2)} \\
& \left.+\gamma_{0} \mathcal{B}^{(2) \dagger} \gamma_{0} \mathcal{C}^{(1)-1} \mathcal{B}^{(1)}+\gamma_{0} \mathcal{B}^{(3) \dagger} \gamma_{0} \mathcal{C}^{(0)-1} \mathcal{B}^{(1)}\right\} H,
\end{aligned}
$$

with

$$
\mathcal{A}^{(3)}=\sum_{i=1}^{218} d_{i} \hat{O}_{i}^{(4)},
$$

where the operators $\hat{O}_{i}^{(4)}$ are compiled in the 3 rd column of Table V.

\section{SUMMARY}

Based on chiral symmetry and basic invariances such as Lorentz invariance, we have constructed the chiral effective Lagrangian for the description of the interactions between the doubly charmed ground-state baryons and Goldstone bosons up to $O\left(q^{4}\right)$. Complete and minimal sets of $O\left(q^{3}\right)$ and $O\left(q^{4}\right)$ operators are established for the first time. The numbers of $O\left(q^{3}\right)$ and $O\left(q^{4}\right)$ terms are 32 and 218, respectively. The involved LECs are expected to be determined by, e.g., experimental or lattice QCD data in the future. The obtained Lagrangian is sufficient for comprehensive analyses of the low-energy physics of the doubly charmed baryons up to the fourth order, enabling us to explore the doubly charmed spectroscopy with high accuracy. Furthermore, it can be readily extended to the sector of doubly bottomed baryons according to heavy quark flavor symmetry.

\section{ACKNOWLEDGMENTS}

We would like to thank Qin-He Yang for useful discussions. This work is supported by National Nature Science Foundations of China (NSFC) under Contract No. 11905258 and by the Fundamental Research Funds for the Central Universities.

\section{APPENDIX A: TYPES OF $\Theta_{\mu \nu \ldots}$}

In this Appendix, a brief introduction to the EOM constraints on the chiral Lagrangian is presented; see Refs. [57,63] for more discussions. $\Gamma$ can be one of the Clifford algebra elements $\left\{1, \gamma_{5} \gamma_{\mu}, \sigma_{\mu \nu}\right\}$ or the Levi-Civita tensor $\epsilon_{\mu \nu \lambda \tau}$.

(i) For the Clifford algebra elements $\left\{1, \gamma_{5} \gamma_{\mu}, \sigma_{\mu \nu}\right\}$, their indices should be distinguished from those of the covariant derivatives that act on the baryon field $\psi$.

(ii) For $\epsilon_{\mu \nu \lambda \tau}$, only one of its indices can be contracted with those of the covariant derivatives that act on the baryon field $\psi$.

Therefore, it is more convenient to use $\Theta_{\mu \nu \ldots}=$ $\left(\Gamma D^{n}\right)_{\mu \nu \ldots}$, taken from Ref. [63], to construct our chiral Lagrangian. The types of $\Theta_{\mu \nu \ldots}$ we need are as follows:

1 $\gamma_{5} \gamma_{\mu}, D_{\mu}$

$$
\begin{aligned}
& \sigma_{\mu \nu}, \gamma_{5} \gamma_{\mu} D_{\nu}, D_{\mu \nu} ; \\
& \gamma_{5} \gamma_{\mu} D_{\nu \lambda}, \sigma_{\mu \nu} D_{\lambda}, \epsilon_{\mu \nu \lambda \rho} D^{\rho}, D_{\mu \nu \lambda} ; \\
& \epsilon_{\mu \nu \lambda \rho}, \gamma_{5} \gamma_{\mu} D_{\nu \lambda \rho}, \sigma_{\mu \nu} D_{\lambda \rho}, \epsilon_{\mu \nu \lambda \tau} D_{\rho}^{\tau}, D_{\mu \nu \lambda \rho} .
\end{aligned}
$$

\section{APPENDIX B: SOME EXPLICIT EXPRESSIONS FOR THE NONRELATIVISTIC LAGRANGIAN}

Basic relations regarding the covariant spin operator $S_{\mu}$ are given below $\left(\epsilon^{0123}=-1\right)$ :

$$
\begin{aligned}
S \cdot v & =0, \quad\left\{S_{\mu}, S_{\nu}\right\}=\frac{1}{2}\left(v_{\mu} v_{\nu}-g_{\mu \nu}\right), \\
{\left[S_{\mu}, S_{\nu}\right] } & =i \epsilon_{\mu \nu \alpha \beta} v^{\alpha} S^{\beta} .
\end{aligned}
$$

By using the above equalities, the Dirac bilinears can be rewritten as

$$
\begin{aligned}
\bar{H} \gamma_{\mu} H & =v_{\mu} \bar{H} H, \quad \bar{h} \gamma_{\mu} H=-2 \bar{h} \gamma_{5} S_{\mu} H, \\
\bar{H} \gamma_{\mu} \gamma_{5} H & =2 \bar{H} S_{\mu} H, \quad \bar{h} \gamma_{\mu} \gamma_{5} H=-v_{\mu} \bar{h} \gamma_{5} H, \\
\bar{H} \sigma^{\mu \nu} H & =-2 i \bar{H}\left[S^{\mu}, S^{\nu}\right] H, \\
\bar{h} \sigma^{\mu \nu} H & =2 i \bar{h} \gamma_{5}\left(v^{\mu} S^{\nu}-v^{\nu} S^{\mu}\right) H .
\end{aligned}
$$

Subsequently, we can readily obtain some relevant explicit expressions of $\mathcal{B}^{(i)}$ and $\mathcal{C}^{(i)}$ : 


$$
\begin{aligned}
& \mathcal{B}^{(1)}=-2 i \gamma_{5} S \cdot D-\frac{g_{A}}{2} \gamma_{5} v \cdot u \\
& \mathcal{B}^{(2)}=-2 b_{7} \gamma_{5}\left(v^{\mu} S^{\nu}-v^{\nu} S^{\mu}\right)\left[u_{\mu}, u_{\nu}\right]+2 i b_{8} \gamma_{5}\left(v^{\mu} S^{\nu}-v^{\nu} S^{\mu}\right) f_{+\mu \nu}, \\
& \mathcal{B}^{(3)}=\gamma_{5}\left(c_{1}\left\{u_{\mu},\left\{u^{\mu}, v \cdot u\right\}\right\}+c_{2}\left[u_{\mu},\left[u^{\mu}, v \cdot u\right]\right]+c_{3} v \cdot u\left\langle u^{2}\right\rangle+c_{4} u_{\mu}\left\langle u^{\mu} v \cdot u\right\rangle-16 m^{2} c_{5}(v \cdot u)^{3}\right. \\
& -4 m^{2} c_{6} v \cdot u\left\langle(v \cdot u)^{2}\right\rangle+c_{15}\left\{v \cdot u, \tilde{\chi}_{+}\right\}+c_{16} v \cdot u\left\langle\chi_{+}\right\rangle+c_{17}\left\langle v \cdot u \tilde{\chi}_{+}\right\rangle+i c_{18}\left[v \cdot D, \tilde{\chi}_{-}\right] \\
& +i c_{19}\left\langle\left[v \cdot D, \chi_{-}\right]\right\rangle+i c_{21}\left[u_{\mu}, f_{+}^{\mu \nu}\right] v_{\nu}+c_{27} \epsilon_{\mu \nu \rho \tau}\left\{u^{\mu}, f_{-}^{\nu \rho}\right\} v^{\tau}+c_{28} \epsilon_{\mu \nu \rho \tau} v^{\tau}\left\langle u^{\mu} f_{-}^{\nu \rho}\right\rangle+c_{31} v_{\nu}\left[D_{\mu}, f_{-}^{\mu \nu}\right] \\
& \left.-4 m^{2} c_{32}\left[v \cdot D, f_{-}^{\mu \nu}\right] v_{\mu} v_{\nu}\right)-2 i \epsilon_{\mu \nu \rho \tau} \gamma_{5} S^{\tau}\left(c_{7}\left\{\left[u^{\mu}, u^{\nu}\right], u^{\rho}\right\}+c_{8}\left\langle\left[u^{\mu}, u^{\nu}\right] u^{\rho}\right\rangle\right) \\
& +4 i m \epsilon_{\mu \nu \lambda \tau} \gamma_{5}\left(v^{\lambda} S^{\tau}-v^{\tau} S^{\lambda}\right)\left\{c_{9}\left\{u^{\mu},\left\{u^{\nu}, v \cdot u\right\}\right\}+c_{10} u^{\mu}\left\langle u^{\nu} v \cdot u\right\rangle\right\} \\
& -2 i c_{11}\left[u_{\mu}, h^{\mu \nu}\right] \gamma_{5} S_{\nu}+8 i m^{2} c_{12} \gamma_{5}\left[S \cdot u, h^{\nu \rho}\right] v_{\nu} v_{\rho}+4 i m \gamma_{5}\left(v_{\mu} S_{\nu}-v_{\nu} S_{\mu}\right) v_{\rho} \\
& \times\left(c_{13}\left\{u^{\mu}, h^{\nu \rho}\right\}+c_{14}\left\langle u^{\mu} h^{\nu \rho}\right\rangle+c_{29}\left\{u^{\mu}, f_{-}^{\nu \rho}\right\}+c_{30}\left\langle u^{\mu} f_{-}^{\nu \rho}\right\rangle\right)-2 c_{20} \gamma_{5}\left[\tilde{\chi}_{-}, S \cdot u\right] \\
& -2 \epsilon_{\mu \nu \rho \tau} \gamma_{5} S^{\tau}\left(c_{22}\left\{u^{\mu}, f_{+}^{\nu \rho}\right\}+c_{23}\left\langle u^{\mu} f_{+}^{\nu \rho}\right\rangle\right)+4 m c_{24} \epsilon_{\nu \rho \lambda \tau}\left[v \cdot u, f_{+}^{\nu \rho}\right] \gamma_{5}\left(v^{\lambda} S^{\tau}-v^{\tau} S^{\lambda}\right)-2 i c_{26}\left[u_{\mu}, f_{-}^{\mu \nu}\right] \gamma_{5} S_{\nu} \text {. } \\
& \mathcal{C}^{(0)}=2 m, \\
& \mathcal{C}^{(1)}=i v \cdot D+g_{A} S \cdot u, \\
& \mathcal{C}^{(2)}=-b_{1}\left\langle\chi_{+}\right\rangle-b_{2} \tilde{\chi}_{+}-b_{3} u^{2}-b_{4}\left\langle u^{2}\right\rangle+8 m^{2} b_{5}(v \cdot u)^{2}+4 m^{2} b_{6}\left\langle(v \cdot u)^{2}\right\rangle-2 b_{7}\left[S^{\mu}, S^{\nu}\right]\left[u_{\mu}, u_{\nu}\right]+2 i b_{8}\left[S_{\mu}, S_{\nu}\right] f_{+}^{\mu \nu} . \\
& \gamma_{0} \mathcal{B}^{(1) \dagger} \gamma_{0} \mathcal{C}^{(0)-1} \mathcal{B}^{(1)}=\frac{2}{m}(S \cdot D)^{2}-\frac{i g_{A}}{2 m}\{S \cdot D, v \cdot u\}-\frac{g_{A}^{2}}{8 m}(v \cdot u)^{2}, \\
& \gamma_{0} \mathcal{B}^{(1) \dagger} \gamma_{0} \mathcal{C}^{(0)-1} \mathcal{B}^{(2)}=-\frac{2 i}{m} b_{7} S \cdot D\left(v^{\mu} S^{\nu}-v^{\nu} S^{\mu}\right)\left[u_{\mu}, u_{\nu}\right]-\frac{2}{m} b_{8} S \cdot D\left(v^{\mu} S^{\nu}-v^{\nu} S^{\mu}\right) f_{+\mu \nu} \\
& +\frac{i g_{A}}{2 m} b_{8}\left(v^{\mu} S^{\nu}-v^{\nu} S^{\mu}\right) v \cdot u f_{+\mu \nu}-\frac{g_{A}}{2 m} b_{7}\left(v^{\mu} S^{\nu}-v^{\nu} S^{\mu}\right) v \cdot u\left[u_{\mu}, u_{\nu}\right], \\
& \gamma_{0} \mathcal{B}^{(1) \dagger} \gamma_{0} \mathcal{C}^{(1)-1} \mathcal{B}^{(1)}=-\frac{i}{m^{2}} S \cdot D v \cdot D S \cdot D-\frac{g_{A}}{4 m^{2}} v \cdot u v \cdot D S \cdot D-\frac{g_{A}}{4 m^{2}} S \cdot D v \cdot D v \cdot u+\frac{i g_{A}^{2}}{16 m^{2}} v \cdot u v \cdot D v \cdot u \\
& -\frac{g_{A}}{m^{2}} S \cdot D S \cdot u S \cdot D+\frac{i g_{A}^{2}}{4 m^{2}} v \cdot u S \cdot u S \cdot D+\frac{i g_{A}^{2}}{4 m^{2}} S \cdot D S \cdot u v \cdot u+\frac{g_{A}^{3}}{16 m^{2}} v \cdot u S \cdot u v \cdot u, \\
& \gamma_{0} \mathcal{B}^{(2) \dagger} \gamma_{0} \mathcal{C}^{(0)-1} \mathcal{B}^{(1)}=\frac{2 i}{m} b_{7}\left(v^{\mu} S^{\nu}-v^{\nu} S^{\mu}\right)\left[u_{\mu}, u_{\nu}\right] S \cdot D+\frac{2}{m} b_{8}\left(v^{\mu} S^{\nu}-v^{\nu} S^{\mu}\right) f_{+\mu \nu} S \cdot D \\
& -\frac{i g_{A}}{2 m} b_{8}\left(v^{\mu} S^{\nu}-v^{\nu} S^{\mu}\right) f_{+\mu \nu} v \cdot u+\frac{g_{A}}{2 m} b_{7}\left(v^{\mu} S^{\nu}-v^{\nu} S^{\mu}\right)\left[u_{\mu}, u_{\nu}\right] v \cdot u,
\end{aligned}
$$




$$
\begin{aligned}
& \gamma_{0} \mathcal{B}^{(1) \dagger} \gamma_{0} \mathcal{C}^{(0)-1} \mathcal{B}^{(3)}=-4 \epsilon_{\mu \nu \lambda \tau} S \cdot D\left(v^{\lambda} S^{\tau}-v^{\tau} S^{\lambda}\right)\left(c_{9}\left\{u^{\mu},\left\{u^{\nu}, v \cdot u\right\}\right\}+c_{10} u^{\mu}\left\langle u^{\nu} v \cdot u\right\rangle\right) \\
& -4 S \cdot D\left(v_{\mu} S_{\nu}-v_{\nu} S_{\mu}\right) v_{\rho}\left(c_{13}\left\{u^{\mu}, h^{\nu \rho}\right\}+c_{14}\left\langle u^{\mu} h^{\nu \rho}\right\rangle+c_{29}\left\{u^{\mu}, f_{-}^{\nu \rho}\right\}\right. \\
& \left.+c_{30}\left\langle u^{\mu} f_{-}^{\nu \rho}\right\rangle\right)+4 i c_{24} \epsilon_{\nu \rho \lambda \tau} S \cdot D\left[v \cdot u, f_{+}^{\nu \rho}\right]\left(v^{\lambda} S^{\tau}-v^{\tau} S^{\lambda}\right) \\
& +\frac{1}{m} S \cdot D\left(i c_{1}\left\{u_{\mu},\left\{u^{\mu}, v \cdot u\right\}\right\}+i c_{2}\left[u_{\mu},\left[u^{\mu}, v \cdot u\right]\right]+2 c_{11}\left[u_{\mu}, h^{\mu \nu}\right] S_{\nu}\right. \\
& +i c_{15}\left\{v \cdot u, \tilde{\chi}_{+}\right\}+i c_{16} v \cdot u\left\langle\chi_{+}\right\rangle+i c_{17}\left\langle v \cdot u \tilde{\chi}_{+}\right\rangle-c_{18}\left[v \cdot D, \tilde{\chi}_{-}\right] \\
& \left.-c_{19}\left\langle\left[v \cdot D, \chi_{-}\right]\right\rangle-2 i c_{20}\left[\tilde{\chi}_{-}, S \cdot u\right]-c_{21}\left[u_{\mu}, f_{+}^{\mu \nu}\right] v_{\nu}+2 c_{26}\left[u_{\mu}, f_{-}^{\mu \nu}\right] S_{\nu}\right) \\
& -\frac{2 i}{m} \epsilon_{\mu \nu \rho \tau} S \cdot D S^{\tau}\left(c_{22}\left\{u^{\mu}, f_{+}^{\nu \rho}\right\}+c_{23}\left\langle u^{\mu} f_{+}^{\nu \rho}\right\rangle\right)+\frac{i}{m} \epsilon_{\mu \nu \rho \tau} S \cdot D \\
& \times\left(c_{27}\left\{u^{\mu}, f_{-}^{\nu \rho}\right\} v^{\tau}+c_{28} v^{\tau}\left\langle u^{\mu} f_{-}^{\nu \rho}\right\rangle\right)+\frac{i}{m} S \cdot D\left(c_{3} v \cdot u\left\langle u^{2}\right\rangle\right. \\
& \left.+c_{31} v_{\nu}\left[D_{\mu}, f_{-}^{\mu \nu}\right]+c_{4} u_{\mu}\left\langle u^{\mu} v \cdot u\right\rangle\right)+\frac{2}{m} c_{7} \epsilon_{\mu \nu \rho \tau} S \cdot D\left\{\left[u^{\mu}, u^{\nu}\right], u^{\rho}\right\} S^{\tau} \\
& +\frac{2}{m} c_{8} \epsilon_{\mu \nu \rho \tau} S \cdot D S^{\tau}\left\langle\left[u^{\mu}, u^{\nu}\right] u^{\rho}\right\rangle-8 m c_{12} S \cdot D\left[S \cdot u, h^{\nu \rho}\right] v_{\nu} v_{\rho} \\
& -4 i m c_{32} S \cdot D\left[v \cdot D, f_{-}^{\mu \nu}\right] v_{\mu} v_{\nu}-4 i m c_{5} S \cdot D\left\{4 c_{5}(v \cdot u)^{3}\right. \\
& \left.+c_{6} v \cdot u\left\langle(v \cdot u)^{2}\right\rangle\right\}+i g_{A} \epsilon_{\mu \nu \lambda \tau}\left(v^{\lambda} S^{\tau}-v^{\tau} S^{\lambda}\right)\left(c_{9} v \cdot u\left\{u^{\mu},\left\{u^{\nu}, v \cdot u\right\}\right\}\right. \\
& \left.+c_{10} v \cdot u u^{\mu}\left\langle u^{\nu} v \cdot u\right\rangle\right)+i g_{A} c_{13} v \cdot u\left(v_{\mu} S_{\nu}-v_{\nu} S_{\mu}\right)\left\{u^{\mu}, h^{\nu \rho}\right\} v_{\rho} \\
& +i g_{A} c_{14} v \cdot u\left(v_{\mu} S_{\nu}-v_{\nu} S_{\mu}\right)\left\langle u^{\mu} h^{\nu \rho}\right\rangle v_{\rho}+g_{A} c_{24} \epsilon_{\nu \rho \lambda \tau} v \cdot u\left[v \cdot u, f_{+}^{\nu \rho}\right] \\
& \times\left(v^{\lambda} S^{\tau}-v^{\tau} S^{\lambda}\right)+i g_{A} c_{29} v \cdot u\left(v_{\mu} S_{\nu}-v_{\mu} S_{\nu}\right) v_{\rho}\left\{u^{\mu}, f_{-}^{\nu \rho}\right\} \\
& +i g_{A} c_{30} v \cdot u\left(v_{\mu} S_{\nu}-v_{\mu} S_{\nu}\right) v_{\rho}\left\langle u^{\mu} f_{-}^{\nu \rho}\right\rangle+\frac{g_{A}}{4 m} c_{1} v \cdot u\left\{u_{\mu},\left\{u^{\mu}, v \cdot u\right\}\right\} \\
& -\frac{i g_{A}}{2 m} c_{11} v \cdot u\left[u_{\mu}, h^{\mu \nu}\right] S_{\nu}+\frac{g_{A}}{4 m} v \cdot u\left(c_{15}\left\{v \cdot u, \tilde{\chi}_{+}\right\}+c_{16} v \cdot u\left\langle\chi_{+}\right\rangle\right. \\
& \left.+c_{17}\left\langle v \cdot u \tilde{\chi}_{+}\right\rangle+i c_{18}\left[v \cdot D, \tilde{\chi}_{-}\right]\right)+\frac{g_{A}}{4 m} c_{2} v \cdot u\left[u_{\mu},\left[u^{\mu}, v \cdot u\right]\right] \\
& +\frac{i g_{A}}{4 m} c_{19} v \cdot u\left\langle\left[v \cdot D, \chi_{-}\right]\right\rangle-\frac{g_{A}}{2 m} c_{20} v \cdot u\left[\tilde{\chi}_{-}, S \cdot u\right] \\
& +\frac{i g_{A}}{4 m} c_{21} v \cdot u\left[u_{\mu}, f_{+}^{\mu \nu}\right] v_{\nu}-\frac{g_{A}}{2 m} \epsilon_{\mu \nu \rho \tau} v \cdot u S^{\tau}\left(c_{22}\left\{u^{\mu}, f_{+}^{\nu \rho}\right\}+c_{23}\left\langle u^{\mu} f_{+}^{\nu \rho}\right\rangle\right) \\
& -\frac{i g_{A}}{2 m} c_{26} v \cdot u\left[u_{\mu}, f_{-}^{\mu \nu}\right] S_{\nu}+\frac{g_{A}}{4 m} c_{27} \epsilon_{\mu \nu \rho \tau} v \cdot u\left\{u^{\mu}, f_{-}^{\nu \rho}\right\} v^{\tau} \\
& +\frac{g_{A}}{4 m} c_{28} \epsilon_{\mu \nu \rho \tau} v \cdot u v^{\tau}\left\langle u^{\mu} f_{-}^{\nu \rho}\right\rangle+\frac{g_{A}}{4 m} c_{3}(v \cdot u)^{2}\left\langle u^{2}\right\rangle \\
& +\frac{g_{A}}{4 m} c_{31} v \cdot u v_{\nu}\left[D_{\mu}, f_{-}^{\mu \nu}\right]+\frac{g_{A}}{4 m} c_{4} v \cdot u u_{\mu}\left\langle u^{\mu} v \cdot u\right\rangle \\
& -\frac{i g_{A}}{2 m} c_{7} \epsilon_{\mu \nu \rho \tau} v \cdot u\left\{\left[u^{\mu}, u^{\nu}\right], u^{\rho}\right\} S^{\tau}-\frac{i g_{A}}{2 m} c_{8} \epsilon_{\mu \nu \rho \tau} v \cdot u S^{\tau}\left\langle\left[u^{\mu}, u^{\nu}\right] u^{\rho}\right\rangle \\
& +2 i m g_{A} c_{12} v \cdot u\left[S \cdot u, h^{\nu \rho}\right] v_{\nu} v_{\rho}-m g_{A} c_{32} v \cdot u\left[v \cdot D, f_{-}^{\mu \nu}\right] v_{\mu} v_{\nu} \\
& -4 m g_{A} c_{5}(v \cdot u)^{4}-m g_{A} c_{6}(v \cdot u)^{2}\left\langle(v \cdot u)^{2}\right\rangle \text {, }
\end{aligned}
$$




$$
\begin{aligned}
\gamma_{0} \mathcal{B}^{(1) \dagger} \gamma_{0} \mathcal{C}^{(1)-1} \mathcal{B}^{(2)}= & -\frac{1}{m^{2}} b_{7} S \cdot D\left(v \cdot D-i g_{A} S \cdot u\right)\left(v^{\mu} S^{\nu}-v^{\nu} S^{\mu}\right)\left[u_{\mu}, u_{\nu}\right] \\
& +\frac{i g_{A}}{4 m^{2}} b_{7} v \cdot u\left(v \cdot D-i g_{A} S \cdot u\right)\left(v^{\mu} S^{\nu}-v^{\nu} S^{\mu}\right)\left[u_{\mu}, u_{\nu}\right] \\
& +\frac{i}{m^{2}} b_{8} S \cdot D\left(v \cdot D-i g_{A} S \cdot u\right)\left(v^{\mu} S^{\nu}-v^{\nu} S^{\mu}\right) f_{+\mu \nu} \\
& +\frac{g_{A}}{4 m^{2}} b_{8} v \cdot u\left(v \cdot D-i g_{A} S \cdot u\right)\left(v^{\mu} S^{\nu}-v^{\nu} S^{\mu}\right) f_{+\mu \nu}
\end{aligned}
$$

$$
\begin{aligned}
\gamma_{0} \mathcal{B}^{(1) \dagger} \gamma_{0} \mathcal{C}^{(2)-1} \mathcal{B}^{(1)}= & -\frac{1}{2 m^{3}} S \cdot D(v \cdot D)^{2} S \cdot D+\frac{i g_{A}}{2 m^{3}} S \cdot D\{v \cdot D, S \cdot u\} S \cdot D \\
& +\frac{g_{A}^{2}}{2 m^{3}} S \cdot D(S \cdot u)^{2} S \cdot D-\frac{1}{m^{2}} S \cdot D C_{(2)} S \cdot D \\
& +\frac{g_{A}^{2}}{32 m^{3}} v \cdot u(v \cdot D)^{2} v \cdot u-\frac{i g_{A}^{3}}{32 m^{3}} v \cdot u\{v \cdot D, S \cdot u\} v \cdot u \\
& -\frac{g_{A}^{4}}{32 m^{3}} v \cdot u(S \cdot u)^{2} v \cdot u+\frac{g_{A}^{2}}{16 m^{2}} v \cdot u C_{(2)} v \cdot u \\
& +\frac{i g_{A}}{8 m^{3}} S \cdot D(v \cdot D)^{2} v \cdot u+\frac{g_{A}^{2}}{8 m^{3}} S \cdot D\{v \cdot D, S \cdot u\} v \cdot u \\
& -\frac{i g_{A}^{3}}{8 m^{3}} S \cdot D(S \cdot u)^{2} v \cdot u+\frac{i g_{A}}{4 m^{2}} S \cdot D C_{(2)} v \cdot u \\
& +\frac{i g_{A}}{8 m^{3}} v \cdot u(v \cdot D)^{2} S \cdot D+\frac{g_{A}^{2}}{8 m^{3}} v \cdot u\{v \cdot D, S \cdot u\} S \cdot D \\
& -\frac{i g_{A}^{3}}{8 m^{3}} v \cdot u(S \cdot u)^{2} S \cdot D+\frac{i g_{A}}{4 m^{2}} v \cdot u C_{(2)} S \cdot D,
\end{aligned}
$$

$$
\begin{aligned}
-\frac{1}{m^{2}} S \cdot D C_{(2)} S \cdot D= & \frac{1}{m^{2}} S \cdot D\left\{b_{1}\left\langle\chi_{+}\right\rangle+b_{2} \tilde{\chi}_{+}+b_{3} u^{2}+b_{4}\left\langle u^{2}\right\rangle+2 b_{7}\left[S^{\mu}, S^{\nu}\right]\left[u_{\mu}, u_{\nu}\right]-2 i b_{8}\left[S_{\mu}, S_{\nu}\right] f_{+}^{\mu \nu}\right\} S \cdot D \\
& -4 S \cdot D\left\{2 b_{5}(v \cdot u)^{2}+b_{6}\left\langle(v \cdot u)^{2}\right\rangle\right\} S \cdot D,
\end{aligned}
$$

$$
\begin{aligned}
\frac{g_{A}^{2}}{16 m^{2}} v \cdot u C_{(2)} v \cdot u= & -\frac{g_{A}^{2}}{16 m^{2}} v \cdot u\left\{b_{1}\left\langle\chi_{+}\right\rangle+b_{2} \tilde{\chi}_{+}+b_{3} u^{2}+b_{4}\left\langle u^{2}\right\rangle+2 b_{7}\left[S^{\mu}, S^{\nu}\right]\left[u_{\mu}, u_{\nu}\right]-2 i b_{8}\left[S_{\mu}, S_{\nu}\right] f_{+}^{\mu \nu}\right\} v \cdot u \\
& +\frac{g_{A}^{2}}{4} v \cdot u\left\{2 b_{5}(v \cdot u)^{2}+b_{6}\left\langle(v \cdot u)^{2}\right\rangle\right\} v \cdot u
\end{aligned}
$$

$$
\begin{aligned}
\frac{i g_{A}}{2 m^{2}} S \cdot D C_{(2)} v \cdot u= & -\frac{i g_{A}}{2 m^{2}} S \cdot D\left\{b_{1}\left\langle\chi_{+}\right\rangle+b_{2} \tilde{\chi}_{+}+b_{3} u^{2}+b_{4}\left\langle u^{2}\right\rangle+2 b_{7}\left[S^{\mu}, S^{\nu}\right]\left[u_{\mu}, u_{\nu}\right]-2 i b_{8}\left[S_{\mu}, S_{\nu}\right] f_{+}^{\mu \nu}\right\} v \cdot u \\
& +2 i g_{A} S \cdot D\left\{2 b_{5}(v \cdot u)^{2}+b_{6}\left\langle(v \cdot u)^{2}\right\rangle\right\} v \cdot u
\end{aligned}
$$

$$
\begin{aligned}
\frac{i g_{A}}{2 m^{2}} v \cdot u C_{(2)} S \cdot D= & -\frac{i g_{A}}{2 m^{2}} v \cdot u\left\{b_{1}\left\langle\chi_{+}\right\rangle+b_{2} \tilde{\chi}_{+}+b_{3} u^{2}+b_{4}\left\langle u^{2}\right\rangle+2 b_{7}\left[S^{\mu}, S^{\nu}\right]\left[u_{\mu}, u_{\nu}\right]-2 i b_{8}\left[S_{\mu}, S_{\nu}\right] f_{+}^{\mu \nu}\right\} S \cdot D \\
& +2 i g_{A} v \cdot u\left\{2 b_{5}(v \cdot u)^{2}+b_{6}\left\langle(v \cdot u)^{2}\right\rangle\right\} S \cdot D
\end{aligned}
$$

$$
\gamma_{0} \mathcal{B}^{(2) \dagger} \gamma_{0} \mathcal{C}^{(0)-1} \mathcal{B}^{(2)}=\frac{2}{m}\left(v^{\mu} S^{\nu}-v^{\nu} S^{\mu}\right)^{2}\left\{b_{7}^{2}\left(\left[u_{\mu}, u_{\nu}\right]\right)^{2}-b_{8}^{2}\left(f_{+\mu \nu}\right)^{2}\right\}-\frac{2 i}{m} b_{7} b_{8}\left(v^{\mu} S^{\nu}-v^{\nu} S^{\mu}\right)^{2}\left\{\left[u_{\mu}, u_{\nu}\right], f_{+}^{\mu \nu}\right\}
$$




$$
\begin{aligned}
\gamma_{0} \mathcal{B}^{(2) \dagger} \gamma_{0} \mathcal{C}^{(1)-1} \mathcal{B}^{(1)}= & \frac{1}{m^{2}} b_{7}\left(v^{\mu} S^{\nu}-v^{\nu} S^{\mu}\right)\left[u_{\mu}, u_{\nu}\right]\left(v \cdot D-i g_{A} S \cdot u\right) S \cdot D \\
& -\frac{i g_{A}}{4 m^{2}} b_{7}\left(v^{\mu} S^{\nu}-v^{\nu} S^{\mu}\right)\left[u_{\mu}, u_{\nu}\right]\left(v \cdot D-i g_{A} S \cdot u\right) v \cdot u \\
& -\frac{i}{m^{2}} b_{8}\left(v^{\mu} S^{\nu}-v^{\nu} S^{\mu}\right) f_{+\mu \nu}\left(v \cdot D-i g_{A} S \cdot u\right) S \cdot D \\
& -\frac{g_{A}}{4 m^{2}} b_{8}\left(v^{\mu} S^{\nu}-v^{\nu} S^{\mu}\right) f_{+\mu \nu}\left(v \cdot D-i g_{A} S \cdot u\right) v \cdot u
\end{aligned}
$$

$$
\begin{aligned}
& \gamma_{0} \mathcal{B}^{(3) \dagger} \gamma_{0} \mathcal{C}^{(0)-1} \mathcal{B}^{(1)}=4 \epsilon_{\mu \nu \lambda \tau}\left(c_{9}\left\{u^{\mu},\left\{u^{\nu}, v \cdot u\right\}\right\}+c_{10} u^{\mu}\left\langle u^{\nu} v \cdot u\right\rangle\right)\left(v^{\lambda} S^{\tau}-v^{\tau} S^{\lambda}\right) S \cdot D+4\left(c_{13}\left\{u^{\mu}, h^{\nu \rho}\right\}\right. \\
& \left.+c_{14}\left\langle u^{\mu} h^{\nu \rho}\right\rangle\right)\left(v_{\mu} S_{\nu}-v_{\nu} S_{\mu}\right) v_{\rho} S \cdot D-4 i c_{24} \epsilon_{\nu \rho \lambda \tau}\left[v \cdot u, f_{+}^{\nu \rho}\right]\left(v^{\lambda} S^{\tau}-v^{\tau} S^{\lambda}\right) S \cdot D \\
& +4\left(c_{29}\left\{u^{\mu}, f_{\underline{\nu}}^{\nu \rho}\right\}+c_{30}\left\langle u^{\mu} f_{-}^{\nu \rho}\right\rangle\right)\left(v_{\mu} S_{\nu}-v_{\mu} S_{\nu}\right) v_{\rho} S \cdot D \\
& +\frac{i}{m}\left(c_{1}\left\{u_{\mu},\left\{u^{\mu}, v \cdot u\right\}\right\}-2 i c_{11}\left[u_{\mu}, h^{\mu \nu}\right] S_{\nu}+c_{15}\left\{v \cdot u, \tilde{\chi}_{+}\right\}\right. \\
& +c_{16} v \cdot u\left\langle\chi_{+}\right\rangle+c_{17}\left\langle v \cdot u \tilde{\chi}_{+}\right\rangle+i c_{18}\left[v \cdot D, \tilde{\chi}_{-}\right]+c_{2}\left[u_{\mu},\left[u^{\mu}, v \cdot u\right]\right] \\
& \left.+i c_{19}\left\langle\left[v \cdot D, \chi_{-}\right]\right\rangle+2 c_{20}\left[\tilde{\chi}_{-}, S \cdot u\right]+i c_{21}\left[u_{\mu}, f_{+}^{\mu \nu}\right] v_{\nu}\right) S \cdot D \\
& -\frac{2 i}{m} \epsilon_{\mu \nu \rho \tau}\left(c_{22}\left\{u^{\mu}, f_{+}^{\nu \rho}\right\}+c_{23}\left\langle u^{\mu} f_{+}^{\nu \rho}\right\rangle\right) S^{\tau} S \cdot D+\frac{2}{m} c_{26}\left[u_{\mu}, f_{-}^{\mu \nu}\right] S_{\nu} S \cdot D-\frac{i}{m} c_{31} v_{\nu}\left[D_{\mu}, f_{-}^{\mu \nu}\right] S \cdot D \\
& +\frac{i}{m} \epsilon_{\mu \nu \rho \tau}\left(c_{27}\left\{u^{\mu}, f_{-}^{\nu \rho}\right\}+c_{28}\left\langle u^{\mu} f_{-}^{\nu \rho}\right\rangle\right) v^{\tau} S \cdot D+\frac{i}{m}\left(c_{3} v \cdot u\left\langle u^{2}\right\rangle+c_{4} u_{\mu}\left\langle u^{\mu} v \cdot u\right\rangle\right) S \cdot D \\
& +\frac{2}{m} \epsilon_{\mu \nu \rho \tau}\left(c_{7}\left\{\left[u^{\mu}, u^{\nu}\right], u^{\rho}\right\}+c_{8}\left\langle\left[u^{\mu}, u^{\nu}\right] u^{\rho}\right\rangle\right) S^{\tau} S \cdot D \\
& -8 m c_{12}\left[S \cdot u, h^{\nu \rho}\right] v_{\nu} v_{\rho} S \cdot D+4 i m c_{32}\left[v \cdot D, f_{-}^{\mu \nu}\right] v_{\mu} v_{\nu} S \cdot D \\
& -4 i m\left(4 c_{5}(v \cdot u)^{3}+c_{6} v \cdot u\left\langle(v \cdot u)^{2}\right\rangle\right) S \cdot D \\
& -i g_{A} \epsilon_{\mu \nu \lambda \tau}\left(c_{9}\left\{u^{\mu},\left\{u^{\nu}, v \cdot u\right\}\right\}+c_{10} u^{\mu}\left\langle u^{\nu} v \cdot u\right\rangle\right)\left(v^{\lambda} S^{\tau}-v^{\tau} S^{\lambda}\right) v \cdot u \\
& -i g_{A}\left(c_{13}\left\{u^{\mu}, h^{\nu \rho}\right\}+c_{14}\left\langle u^{\mu} h^{\nu \rho}\right\rangle\right)\left(v_{\mu} S_{\nu}-v_{\nu} S_{\mu}\right) v_{\rho} v \cdot u-g_{A} c_{24} \epsilon_{\nu \rho \lambda \tau}\left[v \cdot u, f_{+}^{\nu \rho}\right]\left(v^{\lambda} S^{\tau}-v^{\tau} S^{\lambda}\right) v \cdot u \\
& -i g_{A}\left(c_{29}\left\{u^{\mu}, f^{\nu \rho}\right\}+c_{30}\left\langle u^{\mu} f_{-}^{\nu \rho}\right\rangle\right)\left(v_{\mu} S_{\nu}-v_{\mu} S_{\nu}\right) v_{\rho} v \cdot u \\
& +\frac{g_{A}}{4 m}\left(c_{1}\left\{u_{\mu},\left\{u^{\mu}, v \cdot u\right\}\right\}-2 i c_{11}\left[u_{\mu}, h^{\mu \nu}\right] S_{\nu}+c_{15}\left\{v \cdot u, \tilde{\chi}_{+}\right\}\right. \\
& +c_{16} v \cdot u\left\langle\chi_{+}\right\rangle+c_{17}\left\langle v \cdot u \tilde{\chi}_{+}\right\rangle+i c_{18}\left[v \cdot D, \tilde{\chi}_{-}\right]+c_{2}\left[u_{\mu},\left[u^{\mu}, v \cdot u\right]\right] \\
& \left.+i c_{19}\left\langle\left[v \cdot D, \chi_{-}\right]\right\rangle+2 c_{20}\left[\tilde{\chi}_{-}, S \cdot u\right]+i c_{21}\left[u_{\mu}, f_{+}^{\mu \nu}\right] v_{\nu}\right) v \cdot u \\
& -\frac{g_{A}}{2 m} \epsilon_{\mu \nu \rho \tau}\left(c_{22}\left\{u^{\mu}, f_{+}^{\nu \rho}\right\}+c_{23}\left\langle u^{\mu} f_{+}^{\nu \rho}\right\rangle\right) S^{\tau} v \cdot u-\frac{i g_{A}}{2 m} c_{26}\left[u_{\mu}, f_{-}^{\mu \nu}\right] S_{\nu} v \cdot u+\frac{g_{A}}{4 m} c_{3} v \cdot u\left\langle u^{2}\right\rangle v \cdot u \\
& +\frac{g_{A}}{4 m} \epsilon_{\mu \nu \rho \tau}\left(c_{27}\left\{u^{\mu}, f_{-}^{\nu \rho}\right\}+c_{28}\left\langle u^{\mu} f_{-}^{\nu \rho}\right\rangle\right) v^{\tau} v \cdot u-\frac{g_{A}}{4 m} c_{31} v_{\nu}\left[D_{\mu}, f_{-}^{\mu \nu}\right] v \cdot u+\frac{g_{A}}{4 m} c_{4} u_{\mu}\left\langle u^{\mu} v \cdot u\right\rangle v \cdot u \\
& -\frac{i g_{A}}{2 m} \epsilon_{\mu \nu \rho \tau}\left(c_{7}\left\{\left[u^{\mu}, u^{\nu}\right], u^{\rho}\right\}+c_{8}\left\langle\left[u^{\mu}, u^{\nu}\right] u^{\rho}\right\rangle\right) S^{\tau} v \cdot u \\
& +2 i m g_{A} c_{12}\left[S \cdot u, h^{\nu \rho}\right] v_{\nu} v_{\rho} v \cdot u+m g_{A} c_{32}\left[v \cdot D, f_{-}^{\mu \nu}\right] v_{\mu} v_{\nu} v \cdot u \\
& -4 m g_{A} c_{5}(v \cdot u)^{4}-m g_{A} c_{6} v \cdot u\left\langle(v \cdot u)^{2}\right\rangle v \cdot u \text {. }
\end{aligned}
$$




\section{APPENDIX C: THE $\mathcal{O}\left(q^{4}\right)$ OPERATORS}

TABLE V. Terms in the $O\left(q^{4}\right)$ relativistic and nonrelativistic Lagrangians.

\begin{tabular}{|c|c|c|}
\hline$i$ & $O_{i}^{(4)}$ & $\hat{O}_{i}^{(4)}$ \\
\hline 1 & $\left\{u^{\mu},\left\{\left\{u_{\mu}, u^{\nu}\right\}, u_{\nu}\right\}\right\}$ & $\left\{u^{\mu},\left\{\left\{u_{\mu}, u^{\nu}\right\}, u_{\nu}\right\}\right\}$ \\
\hline 2 & $\left\{u^{\mu},\left[\left[u_{\mu}, u^{\nu}\right], u_{\nu}\right]\right\}$ & $\left\{u^{\mu},\left[\left[u_{\mu}, u^{\nu}\right], u_{\nu}\right]\right\}$ \\
\hline 3 & {$\left[u^{\mu},\left\{\left[u_{\mu}, u^{\nu}\right], u_{\nu}\right\}\right]$} & {$\left[u^{\mu},\left\{\left[u_{\mu}, u^{\nu}\right], u_{\nu}\right\}\right]$} \\
\hline 4 & $\left\langle\left\{u^{\mu},\left\{u_{\mu}, u^{\nu}\right\}\right\} u_{\nu}\right\rangle$ & $\left\langle\left\{u^{\mu},\left\{u_{\mu}, u^{\nu}\right\}\right\} u_{\nu}\right\rangle$ \\
\hline 5 & $\left\langle\left[u^{\mu},\left[u_{\mu}, u^{\nu}\right]\right] u_{\nu}\right\rangle$ & $\left\langle\left[u^{\mu},\left[u_{\mu}, u^{\nu}\right]\right] u_{\nu}\right\rangle$ \\
\hline 6 & $u^{\mu}\left\langle u^{2} u_{\mu}\right\rangle$ & $u^{\mu}\left\langle u^{2} u_{\mu}\right\rangle$ \\
\hline 7 & $u^{\mu} u_{\mu}\left\langle u^{2}\right\rangle$ & $u^{2}\left\langle u^{2}\right\rangle$ \\
\hline 8 & $u^{\mu} u^{\nu}\left\langle u_{\mu} u_{\nu}\right\rangle$ & $u^{\mu} u^{\nu}\left\langle u_{\mu} u_{\nu}\right\rangle$ \\
\hline 9 & $i\left\{u^{\mu},\left\{u_{\mu},\left[u^{\nu}, u^{\lambda}\right]\right\}\right\} \sigma_{\nu \lambda}$ & $2\left\{u^{\mu},\left\{u_{\mu},\left[u^{\nu}, u^{\lambda}\right]\right\}\right\}\left[S_{\nu}, S_{\lambda}\right]$ \\
\hline 10 & $i\left[u^{\mu},\left[u_{\mu},\left[u^{\nu}, u^{\lambda}\right]\right]\right] \sigma_{\nu \lambda}$ & $2\left[u^{\mu},\left[u_{\mu},\left[u^{\nu}, u^{\lambda}\right]\right]\right]\left[S_{\nu}, S_{\lambda}\right]$ \\
\hline 11 & $i\left[u^{\nu},\left\{u_{\mu},\left\{u^{\mu}, u^{\lambda}\right\}\right\}\right] \sigma_{\nu \lambda}$ & $2\left[u^{\nu},\left\{u_{\mu},\left\{u^{\mu}, u^{\lambda}\right\}\right\}\right]\left[S_{\nu}, S_{\lambda}\right]$ \\
\hline 12 & $i\left[u^{\nu},\left[u_{\mu},\left[u^{\mu}, u^{\lambda}\right]\right]\right] \sigma_{\nu \lambda}$ & $2\left[u^{\nu},\left[u_{\mu},\left[u^{\mu}, u^{\lambda}\right]\right]\right]\left[S_{\nu}, S_{\lambda}\right]$ \\
\hline 13 & $i\left\langle\left[u_{\mu}, u_{\nu}\right] u^{2}\right\rangle \sigma^{\mu \nu}$ & $2\left[S^{\mu}, S^{\nu}\right]\left\langle\left[u_{\mu}, u_{\nu}\right] u^{2}\right\rangle$ \\
\hline 14 & $i\left\langle\left[u_{\mu},\left\{u^{\lambda}, u_{\nu}\right\}\right] u_{\lambda}\right\rangle \sigma^{\mu \nu}$ & $2\left[S^{\mu}, S^{\nu}\right]\left\langle\left[u_{\mu},\left\{u^{\lambda}, u_{\nu}\right\}\right] u_{\lambda}\right\rangle$ \\
\hline 15 & $i u^{\mu}\left\langle u_{\mu}\left[u_{\nu}, u_{\lambda}\right]\right\rangle \sigma^{\nu \lambda}$ & $2\left[S^{\nu}, S^{\lambda}\right] u^{\mu}\left\langle u_{\mu}\left[u_{\nu}, u_{\lambda}\right]\right\rangle$ \\
\hline 16 & $i\left[u^{\mu}, u^{\nu}\right]\left\langle u^{2}\right\rangle \sigma_{\mu \nu}$ & $2\left[u^{\mu}, u^{\nu}\right]\left[S_{\mu}, S_{\nu}\right]\left\langle u^{2}\right\rangle$ \\
\hline 17 & $i\left[u^{\mu}, u^{\lambda}\right]\left\langle u^{\nu} u_{\lambda}\right\rangle \sigma_{\mu \nu}$ & $2\left[u^{\mu}, u^{\lambda}\right]\left[S_{\mu}, S_{\nu}\right]\left\langle u^{\nu} u_{\lambda}\right\rangle$ \\
\hline 18 & $\left\{u^{\mu},\left\{u_{\mu},\left\{u^{\nu}, u^{\lambda}\right\}\right\}\right\} D_{\nu \lambda}+$ H.c. & $-8 m^{2}\left\{u^{\mu},\left\{u_{\mu},(v \cdot u)^{2}\right\}\right\}$ \\
\hline 19 & {$\left[u^{\mu},\left[u_{\mu},\left\{u^{\nu}, u^{\lambda}\right\}\right]\right] D_{\nu \lambda}+$ H.c. } & $-8 m^{2}\left[u^{\mu},\left[u_{\mu},(v \cdot u)^{2}\right]\right]$ \\
\hline 20 & $\left\{u^{\nu},\left\{u_{\mu},\left\{u^{\mu}, u^{\lambda}\right\}\right\}\right\} D_{\nu \lambda}+$ H.c. & $-4 m^{2}\left\{v \cdot u,\left\{u_{\mu},\left\{u^{\mu}, v \cdot u\right\}\right\}\right\}$ \\
\hline 21 & $\left\{u^{\nu},\left[u_{\mu},\left[u^{\mu}, u^{\lambda}\right]\right]\right\} D_{\nu \lambda}+$ H.c. & $-4 m^{2}\left\{v \cdot u,\left[u_{\mu},\left[u^{\mu}, v \cdot u\right]\right]\right\}$ \\
\hline 22 & $\left\langle\left\{u_{\mu},\left\{u^{\lambda}, u_{\nu}\right\}\right\} u_{\lambda}\right\rangle D^{\mu \nu}+$ H.c. & $-4 m^{2}\left\langle\left\{v \cdot u,\left\{u^{\lambda}, v \cdot u\right\}\right\} u_{\lambda}\right\rangle$ \\
\hline 23 & $\left\langle\left\{u_{\mu}, u_{\nu}\right\} u^{2}\right\rangle D^{\mu \nu}+$ H.c. & $-8 m^{2}\left\langle(v \cdot u)^{2} u^{2}\right\rangle$ \\
\hline 24 & $u^{\mu}\left\langle u_{\nu} u^{2}\right\rangle D_{\mu}^{\nu}+$ H.c. & $-4 m^{2} v \cdot u\left\langle v \cdot u u^{2}\right\rangle$ \\
\hline 25 & $u^{\lambda}\left\langle\left\{u_{\mu}, u_{\nu}\right\} u_{\lambda}\right\rangle D^{\mu \nu}+$ H.c. & $-8 m^{2} u^{\lambda}\left\langle(v \cdot u)^{2} u_{\lambda}\right\rangle$ \\
\hline 26 & $u^{2}\left\langle u_{\nu} u_{\lambda}\right\rangle D^{\nu \lambda}+$ H.c. & $-4 m^{2} u^{2}\left\langle(v \cdot u)^{2}\right\rangle$ \\
\hline 27 & $\left\{u^{\mu}, u^{\nu}\right\}\left\langle u^{2}\right\rangle D_{\mu \nu}+$ H.c. & $-8 m^{2}(v \cdot u)^{2}\left\langle u^{2}\right\rangle$ \\
\hline 28 & $\left\{u^{\mu}, u^{\nu}\right\}\left\langle u_{\nu} u_{\lambda}\right\rangle D_{\mu}^{\lambda}+$ H.c. & $-4 m^{2}\left\{v \cdot u, u^{\nu}\right\}\left\langle u_{\nu} v \cdot u\right\rangle$ \\
\hline 29 & $i\left\{u^{\lambda},\left\{u^{\rho},\left[u^{\mu}, u^{\nu}\right]\right\}\right\} \sigma_{\mu \nu} D_{\lambda \rho}+$ H.c. & $-8 m^{2}\left\{v \cdot u,\left\{v \cdot u,\left[u^{\mu}, u^{\nu}\right]\right\}\right\}\left[S_{\mu}, S_{\nu}\right.$ \\
\hline 30 & $i\left[u^{\lambda},\left[u^{\rho},\left[u^{\mu}, u^{\nu}\right]\right]\right] \sigma_{\mu \nu} D_{\lambda \rho}+$ H.c. & $-8 m^{2}\left[v \cdot u,\left[v \cdot u,\left[u^{\mu}, u^{\nu}\right]\right]\right]\left[S_{\mu}, S_{\nu}\right]$ \\
\hline 31 & $i\left[u^{\mu},\left\{u^{\lambda},\left\{u^{\rho}, u^{\nu}\right\}\right\}\right] \sigma_{\mu \nu} D_{\lambda \rho}+$ H.c. & $-8 m^{2}\left[u^{\mu},\left\{v \cdot u,\left\{v \cdot u, u^{\nu}\right\}\right\}\right]\left[S_{\mu}, S_{\nu}\right.$ \\
\hline 32 & $i\left[u^{\mu},\left[u^{\lambda},\left[u^{\rho}, u^{\nu}\right]\right]\right] \sigma_{\mu \nu} D_{\lambda \rho}+$ H.c. & $-8 m^{2}\left[u^{\mu},\left[v \cdot u,\left[v \cdot u, u^{\nu}\right]\right]\right]\left[S_{\mu}, S_{\nu}\right]$ \\
\hline 33 & $i\left\langle\left\{\left[u_{\mu}, u_{\nu}\right], u_{\lambda}\right\} u_{\rho}\right\rangle \sigma^{\mu \nu} D^{\lambda \rho}+$ H.c. & $-8 m^{2}\left\langle\left\{\left[u_{\mu}, u_{\nu}\right], v \cdot u\right\} v \cdot u\right\rangle\left[S^{\mu}, S^{\nu}\right]$ \\
\hline 34 & $i\left\langle\left[u_{\mu},\left\{u_{\lambda}, u_{\nu}\right\}\right] u_{\rho}\right\rangle \sigma^{\mu \nu} D^{\lambda \rho}+$ H.c. & $-8 m^{2}\left\langle\left[u_{\mu},\left\{v \cdot u, u_{\nu}\right\}\right] v \cdot u\right\rangle\left[S^{\mu}, S^{\nu}\right]$ \\
\hline 35 & $i u^{\mu}\left\langle\left[u_{\nu}, u_{\lambda}\right] u_{\rho}\right\rangle \sigma^{\nu \lambda} D_{\mu}^{\rho}+$ H.c. & $-8 m^{2} v \cdot u\left\langle\left[u_{\nu}, u_{\lambda}\right] v \cdot u\right\rangle\left[S^{\nu}, S^{\lambda}\right]$ \\
\hline 36 & $i\left[u^{\mu}, u^{\nu}\right]\left\langle u_{\lambda} u_{\rho}\right\rangle \sigma_{\mu \nu} D^{\lambda \rho}+$ H.c. & $-8 m^{2}\left[u^{\mu}, u^{\nu}\right]\left[S_{\mu}, S_{\nu}\right]\left\langle(v \cdot u)^{2}\right\rangle$ \\
\hline 37 & $i\left[u^{\mu}, u^{\nu}\right]\left\langle u_{\lambda} u_{\rho}\right\rangle \sigma_{\mu}{ }^{\lambda} D_{\nu}{ }^{\rho}+$ H.c. & $-8 m^{2}\left[u^{\mu}, v \cdot u\right]\left[S_{\mu}, S^{\lambda}\right]\left\langle u_{\lambda} v \cdot u\right\rangle$ \\
\hline 38 & $\left\{u^{\mu},\left\{u^{\nu},\left\{u^{\lambda}, u^{\rho}\right\}\right\}\right\} D_{\mu \nu \lambda \rho}+$ H.c. & $384 m^{4}(v \cdot u)^{4}$ \\
\hline 39 & $\left\langle\left\{u_{\mu},\left\{u_{\nu}, u_{\lambda}\right\}\right\} u_{\rho}\right\rangle D^{\mu \nu \lambda \rho}+$ H.c. & $192 m^{4}\left\langle(v \cdot u)^{4}\right\rangle$ \\
\hline 40 & $u^{\mu}\left\langle\left\{u_{\nu}, u_{\lambda}\right\} u_{\rho}\right\rangle D_{\mu}{ }^{\nu \lambda \rho}+$ H.c. & $96 m^{4} v \cdot u\left\langle(v \cdot u)^{3}\right\rangle$ \\
\hline 41 & $\left\{u^{\mu}, u^{\nu}\right\}\left\langle u_{\lambda} u_{\rho}\right\rangle D_{\mu \nu}^{\lambda \rho}+$ H.c. & $96 m^{4}(v \cdot u)^{2}\left\langle(v \cdot u)^{2}\right\rangle$ \\
\hline 42 & $\epsilon^{\mu \nu \lambda \rho}\left[\left[u_{\mu}, u_{\nu}\right], f_{-\lambda \rho}\right]$ & $\epsilon^{\mu \nu \lambda \rho}\left[\left[u_{\mu}, u_{\nu}\right], f_{-\lambda \rho}\right]$ \\
\hline 43 & $\epsilon^{\mu \nu \lambda \rho} u_{\mu}\left\langle u_{\nu} f_{-\lambda \rho}\right\rangle$ & $\epsilon^{\mu \nu \lambda \rho} u_{\mu}\left\langle u_{\nu} f_{-\lambda \rho}\right\rangle$ \\
\hline 44 & $\left\{\left[u^{\mu}, u^{\nu}\right], f_{-\mu}{ }^{\lambda}\right\} \gamma_{5} \gamma_{\nu} D_{\lambda}+$ H.c. & $4 i m\left\{\left[u^{\mu}, S \cdot u\right], f_{-\mu}^{\lambda}\right\} v_{\lambda}$ \\
\hline 45 & {$\left[\left\{u^{\mu}, u^{\nu}\right\}, f_{-\mu}^{\lambda}\right] \gamma_{5} \gamma_{\nu} D_{\lambda}+$ H.c. } & $4 i m\left[\left\{u^{\mu}, S \cdot u\right\}, f_{-\mu}^{\lambda}\right] v_{\lambda}$ \\
\hline 46 & $\left\{\left[u^{\mu}, u^{\nu}\right], f_{-\mu}^{\lambda}\right\} \gamma_{5} \gamma_{\lambda} D_{\nu}+$ H.c. & $4 \operatorname{im}\left\{\left[u^{\mu}, v \cdot u\right], f_{-\mu}^{\lambda}\right\} S_{\lambda}$ \\
\hline
\end{tabular}


TABLE V. (Continued)

\begin{tabular}{|c|c|c|}
\hline$i$ & $O_{i}^{(4)}$ & $\hat{O}_{i}^{(4)}$ \\
\hline 47 & {$\left[\left\{u^{\mu}, u^{\nu}\right\}, f_{-\mu}{ }^{\lambda}\right] \gamma_{5} \gamma_{\lambda} D_{\nu}+$ H.c. } & $4 i m\left[\left\{u^{\mu}, v \cdot u\right\}, f_{-\mu}^{\lambda}\right] S_{\lambda}$ \\
\hline 48 & $\left\{u^{\mu},\left[u^{\lambda}, f_{-\mu}{ }^{\nu}\right]\right\} \gamma_{5} \gamma_{\nu} D_{\lambda}+$ H.c. & $4 i m\left\{u^{\mu},\left[v \cdot u, f_{-\mu}^{\nu}\right]\right\} S_{\nu}$ \\
\hline 49 & $\left\{u^{\mu},\left[u^{\lambda}, f_{-\mu}^{\nu}\right]\right\} \gamma_{5} \gamma_{\lambda} D_{\nu}+$ H.c. & $4 i m\left\{u^{\mu},\left[S \cdot u, f_{-\mu}^{\nu}\right]\right\} v_{\nu}$ \\
\hline 50 & $\left\{u^{\mu},\left[u_{\mu}, f_{-}^{\nu \lambda}\right]\right\} \gamma_{5} \gamma_{\nu} D_{\lambda}+$ H.c. & $4 i m\left\{u^{\mu},\left[u_{\mu}, f_{-}^{\nu \lambda}\right]\right\} S_{\nu} v_{\lambda}$ \\
\hline 51 & $\left\langle\left[u_{\mu}, u^{\lambda}\right] f_{-\nu \lambda}\right\rangle \gamma_{5} \gamma^{\mu} D^{\nu}+$ H.c. & $4 \operatorname{im}\left\langle\left[S \cdot u, u^{\lambda}\right] f_{-\nu \lambda}\right\rangle v^{\nu}$ \\
\hline 52 & $\left\langle\left[u_{\nu}, u^{\lambda}\right] f_{-\mu \lambda}\right\rangle \gamma_{5} \gamma^{\mu} D^{\nu}+$ H.c. & $4 i m\left\langle\left[v \cdot u, u^{\lambda}\right] f_{-\mu \lambda}\right\rangle S^{\mu}$ \\
\hline 53 & $\left\{u^{\mu},\left[u_{\mu}, h^{\nu \lambda}\right]\right\} \gamma_{5} \gamma_{\nu} D_{\lambda}+$ H.c. & $4 i m\left\{u^{\mu},\left[u_{\mu}, h^{\nu \lambda}\right]\right\} S_{\nu} v_{\lambda}$ \\
\hline 54 & $\left\{\left[u^{\mu}, u^{\nu}\right], h_{\mu}^{\lambda}\right\} \gamma_{5} \gamma_{\nu} D_{\lambda}+$ H.c. & $4 i m\left\{\left[u^{\mu}, S \cdot u\right], h_{\mu}{ }^{\lambda}\right\} v_{\lambda}$ \\
\hline 55 & {$\left[\left\{u^{\mu}, u^{\nu}\right\}, h_{\mu}{ }^{\lambda}\right] \gamma_{5} \gamma_{\nu} D_{\lambda}+$ H.c. } & $4 i m\left[\left\{u^{\mu}, S \cdot u\right\}, h_{\mu}{ }^{\lambda}\right] v_{\lambda}$ \\
\hline 56 & $\left\{\left[u^{\mu}, u^{\nu}\right], h_{\mu}^{\lambda}\right\} \gamma_{5} \gamma_{\lambda} D_{\nu}+$ H.c. & $4 i m\left\{\left[u^{\mu}, v \cdot u\right], h_{\mu}^{\lambda}\right\} S_{\lambda}$ \\
\hline 57 & {$\left[\left\{u^{\mu}, u^{\nu}\right\}, h_{\mu}{ }^{\lambda}\right] \gamma_{5} \gamma_{\lambda} D_{\nu}+$ H.c. } & $4 i m\left[\left\{u^{\mu}, v \cdot u\right\}, h_{\mu}{ }^{\lambda}\right] S_{\lambda}$ \\
\hline 58 & $\left\{u^{\mu},\left[u^{\lambda}, h_{\mu}^{\nu}\right]\right\} \gamma_{5} \gamma_{\nu} D_{\lambda}+$ H.c. & $4 i m\left\{u^{\mu},\left[v \cdot u, h_{\mu}{ }^{\nu}\right]\right\} S_{\nu}$ \\
\hline 59 & $\left\{u^{\mu},\left[u^{\lambda}, h_{\mu}^{\nu}\right]\right\} \gamma_{5} \gamma_{\lambda} D_{\nu}+$ H.c. & $4 i m\left\{u^{\mu},\left[S \cdot u, h_{\mu}{ }^{\nu}\right]\right\} v_{\nu}$ \\
\hline 60 & $\left\langle\left[u_{\mu}, u^{\lambda}\right] h_{\nu \lambda}\right\rangle \gamma_{5} \gamma^{\mu} D^{\nu}+$ H.c. & $4 i m\left\langle\left[S \cdot u, u^{\lambda}\right] h_{\nu \lambda}\right\rangle v^{\nu}$ \\
\hline 61 & $\left\langle\left[u_{\nu}, u^{\lambda}\right] h_{\mu \lambda}\right\rangle \gamma_{5} \gamma^{\mu} D^{\nu}+$ H.c. & $4 i m\left\langle\left[v \cdot u, u^{\lambda}\right] h_{\mu \lambda}\right\rangle S^{\mu}$ \\
\hline 62 & $\epsilon^{\mu \nu \lambda \rho}\left[\left[u_{\mu}, u_{\nu}\right], f_{-\lambda}^{\sigma}\right] D_{\rho \sigma}+$ H.c. & $-4 m^{2} \epsilon^{\mu \nu \lambda \rho}\left[\left[u_{\mu}, u_{\nu}\right], f_{-\lambda}^{\sigma}\right] v_{\rho} v_{\sigma}$ \\
\hline 63 & $\epsilon^{\mu \nu \lambda \rho}\left\{\left\{u_{\mu}, u^{\sigma}\right\}, f_{-\nu \lambda}\right\} D_{\rho \sigma}+$ H.c. & $-4 m^{2} \epsilon^{\mu \nu \lambda \rho}\left\{\left\{u_{\mu}, v \cdot u\right\}, f_{-\nu \lambda}\right\} v_{\rho}$ \\
\hline 64 & $\epsilon^{\mu \nu \lambda \rho}\left[\left[u_{\mu}, u^{\sigma}\right], f_{-\nu \lambda}\right] D_{\rho \sigma}+$ H.c. & $-4 m^{2} \epsilon^{\mu \nu \lambda \rho}\left[\left[u_{\mu}, v \cdot u\right], f_{-\nu \lambda}\right] v_{\rho}$ \\
\hline 65 & $\epsilon^{\mu \nu \lambda \rho}\left\{u_{\mu},\left\{f_{-\nu \lambda}, u^{\sigma}\right\}\right\} D_{\rho \sigma}+$ H.c. & $-4 m^{2} \epsilon^{\mu \nu \lambda \rho}\left\{u_{\mu},\left\{f_{-\nu \lambda}, v \cdot u\right\}\right\} v_{\rho}$ \\
\hline 66 & $\epsilon^{\mu \nu \lambda \rho}\left\langle\left\{u_{\nu}, u_{\sigma}\right\} f_{-\lambda \rho}\right\rangle D_{\mu}^{\sigma}+$ H.c. & $-4 m^{2} \epsilon^{\mu \nu \lambda \rho} v_{\mu}\left\langle\left\{u_{\nu}, v \cdot u\right\} f_{-\lambda \rho}\right\rangle$ \\
\hline 67 & $\epsilon^{\mu \nu \lambda \rho} u_{\mu}\left\langle u_{\nu} f_{-\lambda}{ }^{\sigma}\right\rangle D_{\rho \sigma}+$ H.c. & $-4 m^{2} \epsilon^{\mu \nu \lambda \rho} u_{\mu} v_{\rho} v_{\sigma}\left\langle u_{\nu} f_{-\lambda}{ }^{\sigma}\right\rangle$ \\
\hline 68 & $\epsilon^{\mu \nu \lambda \rho} u_{\mu}\left\langle u^{\sigma} f_{-\nu \lambda}\right\rangle D_{\rho \sigma}+$ H.c. & $-4 m^{2} \epsilon^{\mu \nu \lambda \rho} u_{\mu} v_{\rho}\left\langle v \cdot u f_{-\nu \lambda}\right\rangle$ \\
\hline 69 & $\epsilon^{\mu \nu \lambda \rho} u^{\sigma}\left\langle u_{\mu} f_{-\nu \lambda}\right\rangle D_{\rho \sigma}+$ H.c. & $-4 m^{2} \epsilon^{\mu \nu \lambda \rho} v \cdot u v_{\rho}\left\langle u_{\mu} f_{-\nu \lambda}\right\rangle$ \\
\hline 70 & $\epsilon^{\mu \nu \lambda \rho} f_{-\mu \nu}\left\langle u_{\rho} u_{\sigma}\right\rangle D_{\lambda}^{\sigma}+$ H.c. & $-4 m^{2} \epsilon^{\mu \nu \lambda \rho} f_{-\mu \nu} v_{\lambda}\left\langle u_{\rho} v \cdot u\right\rangle$ \\
\hline 71 & $\epsilon^{\mu \nu \lambda \rho}\left[\left[u_{\mu}, u_{\nu}\right], h_{\lambda}^{\sigma}\right] D_{\rho \sigma}+$ H.c. & $-4 m^{2} \epsilon^{\mu \nu \lambda \rho}\left[\left[u_{\mu}, u_{\nu}\right], h_{\lambda}^{\sigma}\right] v_{\rho} v_{\sigma}$ \\
\hline 72 & $\epsilon^{\mu \nu \lambda \rho} u_{\mu}\left\langle u_{\lambda} h_{\rho \sigma}\right\rangle D_{\nu}^{\sigma}+$ H.c. & $-4 m^{2} \epsilon^{\mu \nu \lambda \rho} u_{\mu} v_{\nu} v^{\sigma}\left\langle u_{\lambda} h_{\rho \sigma}\right\rangle$ \\
\hline 73 & {$\left[\left\{u^{\mu}, u^{\nu}\right\}, f_{-}^{\lambda \rho}\right] \gamma_{5} \gamma_{\lambda} D_{\mu \nu \rho}+$ H.c. } & $-48 \operatorname{im}^{3}\left[(v \cdot u)^{2}, f_{-}^{\lambda \rho}\right] S_{\lambda} v_{\rho}$ \\
\hline 74 & {$\left[\left\{u^{\mu}, u^{\nu}\right\}, h^{\lambda \rho}\right] \gamma_{5} \gamma_{\lambda} D_{\mu \nu \rho}+$ H.c. } & $-48 \operatorname{im}^{3}\left[(v \cdot u)^{2}, h^{\lambda \rho}\right] S_{\lambda} v_{\rho}$ \\
\hline 75 & $\left\{\left[u^{\mu}, u^{\nu}\right], h^{\lambda \rho}\right\} \gamma_{5} \gamma_{\mu} D_{\nu \lambda \rho}+$ H.c. & $-24 i m^{3}\left\{[S \cdot u, v \cdot u], h^{\lambda \rho}\right\} v_{\lambda} v_{\rho}$ \\
\hline 76 & $\left\{u^{\mu},\left[u^{\nu}, h^{\lambda \rho}\right]\right\} \gamma_{5} \gamma_{\mu} D_{\nu \lambda \rho}+$ H.c. & $-24 \operatorname{im}^{3}\left\{S \cdot u,\left[v \cdot u, h^{\lambda \rho}\right]\right\} v_{\lambda} v_{\rho}$ \\
\hline 77 & $\left\{u^{\nu},\left[u^{\mu}, h^{\lambda \rho}\right]\right\} \gamma_{5} \gamma_{\mu} D_{\nu \lambda \rho}+$ H.c. & $-24 \operatorname{im}^{3}\left\{v \cdot u,\left[S \cdot u, h^{\lambda \rho}\right]\right\} v_{\lambda} v_{\rho}$ \\
\hline 78 & $\left\langle\left[u_{\mu}, u_{\nu}\right] h_{\lambda \rho}\right\rangle \gamma_{5} \gamma^{\mu} D^{\nu \lambda \rho}+$ H.c. & $-24 i m^{3}\left\langle[S \cdot u, v \cdot u] h_{\lambda \rho}\right\rangle v_{\lambda} v_{\rho}$ \\
\hline 79 & $i\left\{\left[u_{\mu}, u_{\nu}\right], f_{+}^{\mu \nu}\right\}$ & $i\left\{\left[u_{\mu}, u_{\nu}\right], f_{+}^{\mu \nu}\right\}$ \\
\hline 80 & $i\left\{u_{\mu},\left[u_{\nu}, f_{+}^{\mu \nu}\right]\right\}$ & $i\left\{u_{\mu},\left[u_{\nu}, f_{+}^{\mu \nu}\right]\right\}$ \\
\hline 81 & $i\left\langle\left[u_{\mu}, u_{\nu}\right] f_{+}^{\mu \nu}\right\rangle$ & $i\left\langle\left[u_{\mu}, u_{\nu}\right] f_{+}^{\mu \nu}\right\rangle$ \\
\hline 82 & $\left\{u^{\mu},\left\{u_{\mu}, f_{+}^{\nu \lambda}\right\}\right\} \sigma_{\nu \lambda}$ & $-2 i\left\{u^{\mu},\left\{u_{\mu}, f_{+}^{\nu \lambda}\right\}\right\}\left[S_{\nu}, S_{\lambda}\right]$ \\
\hline 83 & {$\left[u^{\mu},\left[u_{\mu}, f_{+}^{\nu \lambda}\right]\right] \sigma_{\nu \lambda}$} & $-2 i\left[u^{\mu},\left[u_{\mu}, f_{+}^{\nu \lambda}\right]\right]\left[S_{\nu}, S_{\lambda}\right]$ \\
\hline 84 & $\left\{u_{\mu},\left\{u^{\lambda}, f_{+}^{\mu \nu}\right\}\right\} \sigma_{\nu \lambda}$ & $-2 i\left\{u_{\mu},\left\{u^{\lambda}, f_{+}^{\mu \nu}\right\}\right\}\left[S_{\nu}, S_{\lambda}\right]$ \\
\hline 85 & {$\left[u_{\mu},\left[u^{\lambda}, f_{+}^{\mu \nu}\right]\right] \sigma_{\nu \lambda}$} & $-2 i\left[u_{\mu},\left[u^{\lambda}, f_{+}^{\mu \nu}\right]\right]\left[S_{\nu}, S_{\lambda}\right]$ \\
\hline 86 & $\left\{u^{\lambda},\left\{u_{\mu}, f_{+}^{\mu \nu}\right\}\right\} \sigma_{\nu \lambda}$ & $-2 i\left\{u^{\lambda},\left\{u_{\mu}, f_{+}^{\mu \nu}\right\}\right\}\left[S_{\nu}, S_{\lambda}\right]$ \\
\hline 87 & $f_{+}^{\mu \nu}\left\langle u^{2}\right\rangle \sigma_{\mu \nu}$ & $-2 i\left[S_{\mu}, S_{\nu}\right] f_{+}^{\mu \nu}\left\langle u^{2}\right\rangle$ \\
\hline 88 & $u^{\mu}\left\langle f_{+\nu \lambda} u_{\mu}\right\rangle \sigma^{\nu \lambda}$ & $-2 i u^{\mu}\left[S^{\nu}, S^{\lambda}\right]\left\langle f_{+\nu \lambda} u_{\mu}\right\rangle$ \\
\hline 89 & $f_{+}^{\mu \nu}\left\langle u_{\nu} u_{\lambda}\right\rangle \sigma_{\mu}^{\lambda}$ & $-2 i f_{+}^{\mu \nu}\left[S_{\mu}, S^{\lambda}\right]\left\langle u_{\nu} u_{\lambda}\right\rangle$ \\
\hline 90 & $u^{\mu}\left\langle f_{+\nu}^{\lambda} u_{\lambda}\right\rangle \sigma_{\mu}^{\nu}$ & $-2 i u^{\mu}\left[S_{\mu}, S^{\nu}\right]\left\langle f_{+\nu}{ }^{\lambda} u_{\lambda}\right\rangle$ \\
\hline 91 & $u^{\mu}\left\langle f_{+\mu \nu} u_{\lambda}\right\rangle \sigma^{\nu \lambda}$ & $-2 i u^{\mu}\left[S^{\nu}, S^{\lambda}\right]\left\langle f_{+\mu \nu} u_{\lambda}\right\rangle$ \\
\hline 92 & $i\left\{\left[u_{\mu}, u^{\lambda}\right], f_{+}^{\mu \nu}\right\} D_{\nu \lambda}+$ H.c. & $-4 \operatorname{im}^{2}\left\{\left[u_{\mu}, v \cdot u\right], f_{+}^{\mu \nu}\right\} v_{\nu}$ \\
\hline 93 & $i\left[\left\{u_{\mu}, u^{\lambda}\right\}, f_{+}^{\mu \nu}\right] D_{\nu \lambda}+$ H.c. & $-4 \operatorname{im}^{2}\left[\left\{u_{\mu}, v \cdot u\right\}, f_{+}^{\mu \nu}\right] v_{\nu}$ \\
\hline 94 & $i\left\{u_{\mu},\left[u^{\lambda}, f_{+}^{\mu \nu}\right]\right\} D_{\nu \lambda}+$ H.c. & $-4 i m^{2}\left\{u_{\mu},\left[v \cdot u, f_{+}^{\mu \nu}\right]\right\} v_{\nu}$ \\
\hline
\end{tabular}

(Table continued) 
TABLE V. (Continued)

\begin{tabular}{|c|c|c|}
\hline$i$ & $O_{i}^{(4)}$ & $\hat{O}_{i}^{(4)}$ \\
\hline 95 & $i\left\langle\left[u_{\nu}, u_{\lambda}\right] f_{+\mu}{ }^{\lambda}\right\rangle D^{\mu \nu}+$ H.c. & $-4 i m^{2}\left\langle\left[v \cdot u, u_{\lambda}\right] f_{+\mu}^{\lambda}\right\rangle v^{\mu}$ \\
\hline 96 & $\left\{u^{\lambda},\left\{u^{\rho}, f_{+}^{\mu \nu}\right\}\right\} \sigma_{\mu \nu} D_{\lambda \rho}+$ H.c. & $\operatorname{sim}^{2}\left\{v \cdot u,\left\{v \cdot u, f_{+}^{\mu \nu}\right\}\right\}\left[S_{\mu}, S_{\nu}\right]$ \\
\hline 97 & {$\left[u^{\lambda},\left[u^{\rho}, f_{+}^{\mu \nu}\right]\right] \sigma_{\mu \nu} D_{\lambda \rho}+$ H.c. } & $\operatorname{sim}^{2}\left[v \cdot u,\left[v \cdot u, f_{+}^{\mu \nu}\right]\right]\left[S_{\mu}, S_{\nu}\right]$ \\
\hline 98 & $\left\{u^{\lambda},\left\{u^{\rho}, f_{+}^{\mu \nu}\right\}\right\} \sigma_{\mu \lambda} D_{\nu \rho}+$ H.c. & $\operatorname{sim}^{2}\left\{u^{\lambda},\left\{v \cdot u, f_{+}^{\mu \nu}\right\}\right\}\left[S_{\mu}, S_{\lambda}\right] v_{\nu}$ \\
\hline 99 & {$\left[u^{\lambda},\left[u^{\rho}, f_{+}^{\mu \nu}\right]\right] \sigma_{\mu \lambda} D_{\nu \rho}+$ H.c. } & $\operatorname{sim}^{2}\left[u^{\lambda},\left[v \cdot u, f_{+}^{\mu \nu}\right]\right]\left[S_{\mu}, S_{\lambda}\right] v_{\nu}$ \\
\hline 100 & $\left\{u^{\rho},\left\{u^{\lambda}, f_{+}^{\mu \nu}\right\}\right\} \sigma_{\mu \lambda} D_{\nu \rho}+$ H.c. & $\operatorname{sim}^{2}\left\{v \cdot u,\left\{u^{\lambda}, f_{+}^{\mu \nu}\right\}\right\}\left[S_{\mu}, S_{\lambda}\right] v_{\nu}$ \\
\hline 101 & $f_{+}^{\mu \nu}\left\langle u_{\lambda} u_{\rho}\right\rangle \sigma_{\mu \nu} D^{\lambda \rho}+$ H.c. & $\operatorname{8im}^{2} f_{+}^{\mu \nu}\left[S_{\mu}, S_{\nu}\right]\left\langle(v \cdot u)^{2}\right\rangle$ \\
\hline 102 & $f_{+}^{\mu \nu}\left\langle u_{\lambda} u_{\rho}\right\rangle \sigma_{\mu}^{\lambda} D_{\nu}^{\rho}+$ H.c. & $\operatorname{8im}^{2} f_{+}^{\mu \nu}\left[S_{\mu}, S^{\lambda}\right] v_{\nu}\left\langle u_{\lambda} v \cdot u\right\rangle$ \\
\hline 103 & $u^{\mu}\left\langle f_{+\nu \lambda} u_{\rho}\right\rangle \sigma_{\mu}^{\nu} D^{\lambda \rho}+$ H.c. & $8 i m^{2} u^{\mu}\left[S_{\mu}, S^{\nu}\right]\left\langle f_{+\nu \lambda} v \cdot u\right\rangle v^{\lambda}$ \\
\hline 104 & $u^{\mu}\left\langle f_{+\nu \lambda} u_{\rho}\right\rangle \sigma^{\nu \lambda} D_{\mu}^{\rho}+$ H.c. & $8 \operatorname{sim}^{2} v \cdot u\left[S^{\nu}, S^{\lambda}\right]\left\langle f_{+\nu \lambda} v \cdot u\right\rangle$ \\
\hline 105 & $u^{\mu}\left\langle f_{+\nu \rho} u_{\lambda}\right\rangle \sigma^{\nu \lambda} D_{\mu}{ }^{\rho}+$ H.c. & $8 i m^{2} v \cdot u\left[S^{\nu}, S^{\lambda}\right]\left\langle f_{+\nu \rho} u_{\lambda}\right\rangle v^{\rho}$ \\
\hline 106 & $i\left\{\left[u^{\mu}, u^{\nu}\right], \tilde{\chi}_{-}\right\} \gamma_{5} \gamma_{\mu} D_{\nu}+$ H.c. & $-4 m\left\{[S \cdot u, v \cdot u], \tilde{\chi}_{-}\right\}$ \\
\hline 107 & $i\left[\left\{u^{\mu}, u^{\nu}\right\}, \tilde{\chi}_{-}\right] \gamma_{5} \gamma_{\mu} D_{\nu}+$ H.c. & $-4 m\left[\{S \cdot u, v \cdot u\}, \tilde{\chi}_{-}\right]$ \\
\hline 108 & $i\left\{u^{\mu},\left[u^{\nu}, \tilde{\chi}_{-}\right]\right\} \gamma_{5} \gamma_{\mu} D_{\nu}+$ H.c. & $-4 m\left\{S \cdot u,\left[v \cdot u, \tilde{\chi}_{-}\right]\right\}$ \\
\hline 109 & $i\left\langle\left[u_{\mu}, u_{\nu}\right] \tilde{\chi}_{-}\right\rangle \gamma_{5} \gamma^{\mu} D^{\nu}+$ H.c. & $-4 m\left\langle[S \cdot u, v \cdot u] \tilde{\chi}_{-}\right\rangle$ \\
\hline 110 & $i\left[u_{\mu}, u_{\nu}\right]\left\langle\chi_{-}\right\rangle \gamma_{5} \gamma^{\mu} D^{\nu}+$ H.c. & $-4 m[S \cdot u, v \cdot u]\left\langle\chi_{-}\right\rangle$ \\
\hline 111 & $\left\{u_{\mu},\left\{u^{\mu}, \tilde{\chi}_{+}\right\}\right\}$ & $\left\{u_{\mu},\left\{u^{\mu}, \tilde{\chi}_{+}\right\}\right\}$ \\
\hline 112 & {$\left[u_{\mu},\left[u^{\mu}, \tilde{\chi}_{+}\right]\right]$} & {$\left[u_{\mu},\left[u^{\mu}, \tilde{\chi}_{+}\right]\right]$} \\
\hline 113 & $u^{\mu}\left\langle u_{\mu} \tilde{\chi}_{+}\right\rangle$ & $u^{\mu}\left\langle u_{\mu} \tilde{\chi}_{+}\right\rangle$ \\
\hline 114 & $\tilde{\chi}_{+}\left\langle u^{2}\right\rangle$ & $\tilde{\chi}_{+}\left\langle u^{2}\right\rangle$ \\
\hline 115 & $u^{2}\left\langle\chi_{+}\right\rangle$ & $u^{2}\left\langle\chi_{+}\right\rangle$ \\
\hline 116 & $i\left\{u^{\mu},\left[u^{\nu}, \tilde{\chi}_{+}\right]\right\} \sigma_{\mu \nu}$ & $2\left\{u^{\mu},\left[u^{\nu}, \tilde{\chi}_{+}\right]\right\}\left[S_{\mu}, S_{\nu}\right]$ \\
\hline 117 & $i\left[u^{\mu},\left\{u^{\nu}, \tilde{\chi}_{+}\right\}\right] \sigma_{\mu \nu}$ & $2\left[u^{\mu},\left\{u^{\nu}, \tilde{\chi}_{+}\right\}\right]\left[S_{\mu}, S_{\nu}\right]$ \\
\hline 118 & $i\left\langle\left[u_{\mu}, u_{\nu}\right] \tilde{\chi}_{+}\right\rangle \sigma^{\mu \nu}$ & $2\left[S^{\mu}, S^{\nu}\right]\left\langle\left[u_{\mu}, u_{\nu}\right] \tilde{\chi}_{+}\right\rangle$ \\
\hline 119 & $i\left[u^{\mu}, u^{\nu}\right]\left\langle\chi_{+}\right\rangle \sigma_{\mu \nu}$ & $2\left[u^{\mu}, u^{\nu}\right]\left[S_{\mu}, S_{\nu}\right]\left\langle\chi_{+}\right\rangle$ \\
\hline 120 & $\left\{u^{\mu},\left\{u^{\nu}, \tilde{\chi}_{+}\right\}\right\} D_{\mu \nu}+$ H.c. & $-4 m^{2}\left\{v \cdot u,\left\{v \cdot u, \tilde{\chi}_{+}\right\}\right\}$ \\
\hline 121 & {$\left[u^{\mu},\left[u^{\nu}, \tilde{\chi}_{+}\right]\right] D_{\mu \nu}+$ H.c. } & $-4 m^{2}\left[v \cdot u,\left[v \cdot u, \tilde{\chi}_{+}\right]\right]$ \\
\hline 122 & $u^{\mu}\left\langle u_{\nu} \tilde{\chi}_{+}\right\rangle D_{\mu}^{\nu}+$ H.c. & $-4 m^{2} v \cdot u\left\langle v \cdot u \tilde{\chi}_{+}\right\rangle$ \\
\hline 123 & $\tilde{\chi}_{+}\left\langle u_{\mu} u_{\nu}\right\rangle D^{\mu \nu}+$ H.c. & $-4 m^{2} \tilde{\chi}_{+}\left\langle(v \cdot u)^{2}\right\rangle$ \\
\hline 124 & $\left\{u^{\mu}, u^{\nu}\right\}\left\langle\chi_{+}\right\rangle D_{\mu \nu}+$ H.c. & $-8 m^{2}(v \cdot u)^{2}\left\langle\chi_{+}\right\rangle$ \\
\hline 125 & {$\left[u^{\mu},\left[D^{\nu}, \tilde{\chi}_{+}\right]\right] \gamma_{5} \gamma_{\nu} D_{\mu}+$ H.c. } & $4 i m\left[v \cdot u,\left[S \cdot D, \tilde{\chi}_{+}\right]\right]$ \\
\hline 126 & {$\left[u^{\mu},\left[D^{\nu}, \tilde{\chi}_{+}\right]\right] \gamma_{5} \gamma_{\mu} D_{\nu}+$ H.c. } & $4 i m\left[S \cdot u,\left[v \cdot D, \tilde{\chi}_{+}\right]\right]$ \\
\hline 127 & $i\left\{u^{\mu},\left[D_{\mu}, \tilde{\chi}_{-}\right]\right\}$ & $i\left\{u^{\mu},\left[D_{\mu}, \tilde{\chi}_{-}\right]\right\}$ \\
\hline 128 & $i\left\langle u^{\mu}\left[D_{\mu}, \tilde{\chi}_{-}\right]\right\rangle$ & $i\left\langle u^{\mu}\left[D_{\mu}, \tilde{\chi}_{-}\right]\right\rangle$ \\
\hline 129 & $i u^{\mu}\left\langle\left[D_{\mu}, \chi_{-}\right]\right\rangle$ & $i u^{\mu}\left\langle\left[D_{\mu}, \chi_{-}\right]\right\rangle$ \\
\hline 130 & {$\left[u^{\mu},\left[D^{\nu}, \tilde{\chi}_{-}\right]\right] \sigma_{\mu \nu}$} & $-2 i\left[u^{\mu},\left[D^{\nu}, \tilde{\chi}_{-}\right]\right]\left[S_{\mu}, S_{\nu}\right]$ \\
\hline 131 & $i\left\{u^{\mu},\left[D^{\nu}, \tilde{\chi}_{-}\right]\right\} D_{\mu \nu}+$ H.c. & $-4 \operatorname{im}^{2}\left\{v \cdot u,\left[v \cdot D, \tilde{\chi}_{-}\right]\right\}$ \\
\hline 132 & $i\left\langle u^{\mu}\left[D^{\nu}, \tilde{\chi}_{-}\right]\right\rangle D_{\mu \nu}+$ H.c. & $-4 i m^{2}\left\langle v \cdot u\left[v \cdot D, \tilde{\chi}_{-}\right]\right\rangle$ \\
\hline 133 & $i u^{\mu}\left\langle\left[D_{\nu}, \chi_{-}\right]\right\rangle D_{\mu}{ }^{\nu}+$ H.c. & $-4 i m^{2} v \cdot u\left\langle\left[v \cdot D, \chi_{-}\right]\right\rangle$ \\
\hline 134 & $i\left\{\left[D^{\mu}, f_{+\mu}^{\nu}\right], u^{\lambda}\right\} \gamma_{5} \gamma_{\nu} D_{\lambda}+$ H.c. & $-4 m\left\{\left[D^{\mu}, f_{+\mu}^{\nu}\right], v \cdot u\right\} S_{\nu}$ \\
\hline 135 & $i\left\{\left[D^{\mu}, f_{+\mu}^{\nu}\right], u^{\lambda}\right\} \gamma_{5} \gamma_{\lambda} D_{\nu}+$ H.c. & $-4 m\left\{\left[D^{\mu}, f_{+\mu}{ }^{\nu}\right], S \cdot u\right\} v_{\nu}$ \\
\hline 136 & $i\left\{\left[D^{\mu}, f_{+\lambda}^{\nu}\right], u^{\lambda}\right\} \gamma_{5} \gamma_{\mu} D_{\nu}+$ H.c. & $-4 m\left\{\left[S \cdot D, f_{+\lambda^{\nu}}\right], u^{\lambda}\right\} v_{\nu}$ \\
\hline 137 & $i\left\{\left[D^{\mu}, f_{+}^{\nu \lambda}\right], u_{\mu}\right\} \gamma_{5} \gamma_{\nu} D_{\lambda}+$ H.c. & $-4 m\left\{\left[D^{\mu}, f_{+}^{\nu \lambda}\right], u_{\mu}\right\} S_{\nu} v_{\lambda}$ \\
\hline 138 & $i\left\langle\left[D_{\mu}, f_{+\nu}{ }^{\lambda}\right] u_{\lambda}\right\rangle \gamma_{5} \gamma^{\mu} D^{\nu}+$ H.c. & $-4 m\left\langle\left[S \cdot D, f_{+\nu}^{\lambda}\right] u_{\lambda}\right\rangle v_{\nu}$ \\
\hline 139 & $i\left\langle\left[D^{\lambda}, f_{+\mu \lambda}\right] u_{\nu}\right\rangle \gamma_{5} \gamma^{\mu} D^{\nu}+$ H.c. & $-4 m S^{\mu}\left\langle\left[D^{\lambda}, f_{+\mu \lambda}\right] v \cdot u\right\rangle$ \\
\hline 140 & $i\left\langle\left[D^{\mu}, f_{+}^{\nu \lambda}\right] u_{\mu}\right\rangle \gamma_{5} \gamma_{\nu} D_{\lambda}+$ H.c. & $-4 m\left\langle\left[D^{\mu}, f_{+}^{\nu \lambda}\right] u_{\mu}\right\rangle S_{\nu} v_{\lambda}$ \\
\hline 141 & $i\left\langle\left[D^{\lambda}, f_{+\nu \lambda}\right] u_{\mu}\right\rangle \gamma_{5} \gamma^{\mu} D^{\nu}+$ H.c. & $-4 m\left\langle\left[D^{\lambda}, f_{+\nu \lambda}\right] S \cdot u\right\rangle v^{\nu}$ \\
\hline 142 & $\left\{u^{\mu},\left[D^{\nu}, f_{-\mu \nu}\right]\right\}$ & $\left\{u^{\mu},\left[D^{\nu}, f_{-\mu \nu}\right]\right\}$ \\
\hline 143 & $\left\langle u^{\mu}\left[D^{\nu}, f_{-\mu \nu}\right]\right\rangle$ & $\left\langle u^{\mu}\left[D^{\nu}, f_{-\mu \nu}\right]\right\rangle$ \\
\hline 144 & $i\left[u^{\mu},\left[D_{\mu}, f_{-}^{\nu \lambda}\right]\right] \sigma_{\nu \lambda}$ & $2\left[u^{\mu},\left[D_{\mu}, f_{-}^{\nu \lambda}\right]\right]\left[S_{\nu}, S_{\lambda}\right]$ \\
\hline
\end{tabular}


TABLE V. (Continued)

\begin{tabular}{|c|c|c|}
\hline$i$ & $O_{i}^{(4)}$ & $\hat{O}_{i}^{(4)}$ \\
\hline 145 & $i\left[u^{\mu},\left[D^{\nu}, f_{-\nu}{ }^{\lambda}\right]\right] \sigma_{\mu \lambda}$ & $2\left[u^{\mu},\left[D^{\nu}, f_{-\nu}{ }^{\lambda}\right]\right]\left[S_{\mu}, S_{\lambda}\right]$ \\
\hline 146 & $i\left[u_{\nu},\left[D^{\mu}, f_{-}^{\nu \lambda}\right]\right] \sigma_{\mu \lambda}$ & $2\left[u_{\nu},\left[D^{\mu}, f_{-}^{\nu \lambda}\right]\right]\left[S_{\mu}, S_{\lambda}\right]$ \\
\hline 147 & $\left\{u^{\lambda},\left[D_{\mu}, f_{-}^{\mu \nu}\right]\right\} D_{\nu \lambda}+$ H.c. & $-4 m^{2}\left\{v \cdot u,\left[D_{\mu}, f_{-}^{\mu \nu}\right]\right\} v_{\nu}$ \\
\hline 148 & $\left\{u_{\mu},\left[D^{\lambda}, f_{-}^{\mu \nu}\right]\right\} D_{\nu \lambda}+$ H.c. & $-4 m^{2}\left\{u_{\mu},\left[v \cdot D, f_{-}^{\mu \nu}\right]\right\} v_{\nu}$ \\
\hline 149 & $\left\langle u_{\mu}\left[D^{\lambda}, f_{-}^{\mu \nu}\right]\right\rangle D_{\nu \lambda}+$ H.c. & $-4 m^{2} v_{\nu}\left\langle u_{\mu}\left[v \cdot D, f_{-}^{\mu \nu}\right]\right\rangle$ \\
\hline 150 & $\left\langle u^{\lambda}\left[D_{\mu}, f_{-}^{\mu \nu}\right]\right\rangle D_{\nu \lambda}+$ H.c. & $-4 m^{2} v_{\nu}\left\langle v \cdot u\left[D_{\mu}, f_{-}^{\mu \nu}\right]\right\rangle$ \\
\hline 151 & $\tilde{\chi}_{+}^{2}$ & $\tilde{\chi}_{+}^{2}$ \\
\hline 152 & $\left\langle\tilde{\chi}_{+}^{2}\right\rangle$ & $\left\langle\tilde{\chi}_{+}^{2}\right\rangle$ \\
\hline 153 & $\tilde{\chi}_{+}\left\langle\chi_{+}\right\rangle$ & $\tilde{\chi}_{+}\left\langle\chi_{+}\right\rangle$ \\
\hline 154 & $\left\langle\chi_{+}\right\rangle^{2}$ & $\left\langle\chi_{+}\right\rangle^{2}$ \\
\hline 155 & $\left\{f_{+}^{\mu \nu}, \tilde{\chi}_{+}\right\} \sigma_{\mu \nu}$ & $-2 i\left\{f_{+}^{\mu \nu}, \tilde{\chi}_{+}\right\}\left[S_{\mu}, S_{\nu}\right]$ \\
\hline 156 & $\left\langle f_{+\mu \nu} \tilde{\chi}_{+}\right\rangle \sigma^{\mu \nu}$ & $-2 i\left[S^{\mu}, S^{\nu}\right]\left\langle f_{+\mu \nu} \tilde{\chi}_{+}\right\rangle$ \\
\hline 157 & $f_{+}^{\mu \nu}\left\langle\chi_{+}\right\rangle \sigma_{\mu \nu}$ & $-2 i f_{+}^{\mu \nu}\left[S_{\mu}, S_{\nu}\right]\left\langle\chi_{+}\right\rangle$ \\
\hline 158 & {$\left[\tilde{\chi}_{+}, h^{\mu \nu}\right] \gamma_{5} \gamma_{\mu} D_{\nu}+$ H.c. } & $4 i m\left[\tilde{\chi}_{+}, h^{\mu \nu}\right] S_{\mu} v_{\nu}$ \\
\hline 159 & {$\left[\tilde{\chi}_{+}, f_{-}^{\mu \nu}\right] \gamma_{5} \gamma_{\mu} D_{\nu}+$ H.c. } & $4 i m\left[\tilde{\chi}_{+}, f_{-}^{\mu \nu}\right] S_{\mu} v_{\nu}$ \\
\hline 160 & {$\left[D^{2}, \tilde{\chi}_{+}\right]$} & {$\left[D^{2}, \tilde{\chi}_{+}\right]$} \\
\hline 161 & $\left\langle\left[D^{2}, \chi_{+}\right]\right\rangle$ & $\left\langle\left[D^{2}, \chi_{+}\right]\right\rangle$ \\
\hline 162 & $\tilde{\chi}_{-}^{2}$ & $\tilde{\chi}_{-}^{2}$ \\
\hline 163 & $\left\langle\tilde{\chi}_{-}^{2}\right\rangle$ & $\left\langle\tilde{\chi}_{-}^{2}\right\rangle$ \\
\hline 164 & $\tilde{\chi}_{-}\left\langle\chi_{-}\right\rangle$ & $\tilde{\chi}_{-}\left\langle\chi_{-}\right\rangle$ \\
\hline 165 & $\left\langle\chi_{-}\right\rangle^{2}$ & $\left\langle\chi_{-}\right\rangle^{2}$ \\
\hline 166 & $\left\{f_{+}^{\mu \nu}, \tilde{\chi}_{-}\right\} \gamma_{5} \gamma_{\mu} D_{\nu}+$ H.c. & $4 i m\left\{f_{+}^{\mu \nu}, \tilde{\chi}_{-}\right\} S_{\mu} v_{\nu}$ \\
\hline 167 & $\left\langle f_{+}^{\mu \nu} \tilde{\chi}_{-}\right\rangle \gamma_{5} \gamma_{\mu} D_{\nu}+$ H.c. & $4 i m\left\langle f_{+}^{\mu \nu} \tilde{\chi}_{-}\right\rangle S_{\mu} v_{\nu}$ \\
\hline 168 & $f_{+}^{\mu \nu}\left\langle\chi_{-}\right\rangle \gamma_{5} \gamma_{\mu} D_{\nu}+$ H.c. & $4 i m f_{+}^{\mu \nu}\left\langle\chi_{-}\right\rangle S_{\mu} v_{\nu}$ \\
\hline 169 & $i\left\{h^{\mu \nu}, \tilde{\chi}_{-}\right\} D_{\mu \nu}+$ H.c. & $-4 i m^{2}\left\{h^{\mu \nu}, \tilde{\chi}_{-}\right\} v_{\mu} v_{\nu}$ \\
\hline 170 & $i\left\langle h_{\mu \nu} \tilde{\chi}_{-}\right\rangle D^{\mu \nu}+$ H.c. & $-4 i m^{2} v^{\mu} v^{\nu}\left\langle h_{\mu \nu} \tilde{\chi}_{-}\right\rangle$ \\
\hline 171 & $i h_{\mu \nu}\left\langle\chi_{-}\right\rangle D^{\mu \nu}+$ H.c. & $-4 i m^{2} h_{\mu \nu} v^{\mu} v^{\nu}\left\langle\chi_{-}\right\rangle$ \\
\hline 172 & {$\left[f_{-}^{\mu \nu}, \tilde{\chi}_{-}\right] \sigma_{\mu \nu}$} & $-2 i\left[f_{-}^{\mu \nu}, \tilde{\chi}_{-}\right]\left[S_{\mu}, S_{\nu}\right]$ \\
\hline 173 & $\left\{f_{+}^{\mu \nu}, f_{+\mu \nu}\right\}$ & $\left\{f_{+}^{\mu \nu}, f_{+\mu \nu}\right\}$ \\
\hline 174 & $\left\langle f_{+}^{\mu \nu} f_{+\mu \nu}\right\rangle$ & $\left\langle f_{+}^{\mu \nu} f_{+\mu \nu}\right\rangle$ \\
\hline 175 & $i\left[f_{+}^{\mu \nu}, f_{+\mu}^{\lambda}\right] \sigma_{\nu \lambda}$ & $2\left[f_{+}^{\mu \nu}, f_{+\mu}^{\lambda}\right]\left[S_{\nu}, S_{\lambda}\right]$ \\
\hline 176 & $\left\{f_{+}^{\mu \nu}, f_{+\mu}{ }^{\lambda}\right\} D_{\nu \lambda}+$ H.c. & $-4 m^{2}\left\{f_{+}^{\mu \nu}, f_{+\mu}^{\lambda}\right\} v_{\nu} v_{\lambda}$ \\
\hline 177 & $\left\langle f_{+\mu}^{\lambda} f_{+\nu \lambda}\right\rangle D^{\mu \nu}+$ H.c. & $-4 m^{2} v^{\mu} v^{\nu}\left\langle f_{+\mu}^{\lambda} f_{+\nu \lambda}\right\rangle$ \\
\hline 178 & $i\left[f_{+}^{\mu \nu}, f_{+}^{\lambda \rho}\right] \sigma_{\mu \lambda} D_{\nu \rho}+$ H.c. & $-8 m^{2}\left[f_{+}^{\mu \nu}, f_{+}^{\lambda \rho}\right]\left[S_{\mu}, S_{\lambda}\right] v_{\nu} v_{\rho}$ \\
\hline 179 & $i\left\{f_{+}^{\mu \nu}, h_{\mu}{ }^{\lambda}\right\} \gamma_{5} \gamma_{\nu} D_{\lambda}+$ H.c. & $-4 m\left\{f_{+}^{\mu \nu}, h_{\mu}^{\lambda}\right\} S_{\nu} v_{\lambda}$ \\
\hline 180 & $i\left\{f_{+}^{\mu \nu}, h_{\mu}^{\lambda}\right\} \gamma_{5} \gamma_{\lambda} D_{\nu}+$ H.c. & $-4 m\left\{f_{+}^{\mu \nu}, h_{\mu}^{\lambda}\right\} S_{\lambda} v_{\nu}$ \\
\hline 181 & $i\left\langle f_{+\mu}{ }^{\lambda} h_{\nu \lambda}\right\rangle \gamma_{5} \gamma^{\mu} D^{\nu}+$ H.c. & $-4 m S^{\mu} v^{\nu}\left\langle f_{+\mu}^{\lambda} h_{\nu \lambda}\right\rangle$ \\
\hline 182 & $i\left\langle f_{+\nu}{ }^{\lambda} h_{\mu \lambda}\right\rangle \gamma_{5} \gamma^{\mu} D^{\nu}+$ H.c. & $-4 m S^{\mu} v^{\nu}\left\langle f_{+\nu}^{\lambda} h_{\mu \lambda}\right\rangle$ \\
\hline 183 & $i \epsilon^{\mu \nu \lambda \rho}\left[f_{+\mu \nu}, h_{\lambda}^{\sigma}\right] D_{\rho \sigma}+$ H.c. & $-4 \operatorname{im}^{2} \epsilon^{\mu \nu \lambda \rho}\left[f_{+\mu \nu}, h_{\lambda}^{\sigma}\right] v_{\rho} v_{\sigma}$ \\
\hline 184 & $i\left\{f_{+}^{\mu \nu}, h^{\lambda \rho}\right\} \gamma_{5} \gamma_{\mu} D_{\nu \lambda \rho}+$ H.c. & $24 m^{3}\left\{f_{+}^{\mu \nu}, h^{\lambda \rho}\right\} S_{\mu} v_{\nu} v_{\lambda} v_{\rho}$ \\
\hline 185 & $i\left\langle f_{+\mu \nu} h_{\lambda \rho}\right\rangle \gamma_{5} \gamma^{\mu} D^{\nu \lambda \rho}+$ H.c. & $24 m^{3} S^{\mu} v^{\nu} v^{\lambda} v^{\rho}\left\langle f_{+\mu \nu} h_{\lambda \rho}\right\rangle$ \\
\hline 186 & $i \epsilon^{\mu \nu \lambda \rho}\left[f_{+\mu \nu}, f_{-\lambda \rho}\right]$ & $i \epsilon^{\mu \nu \lambda \rho}\left[f_{+\mu \nu}, f_{-\lambda \rho}\right]$ \\
\hline 187 & $i\left\{f_{+}^{\mu \nu}, f_{-\mu}^{\lambda}\right\} \gamma_{5} \gamma_{\nu} D_{\lambda}+$ H.c. & $-4 m S_{\nu} v_{\lambda}\left\{f_{+}^{\mu \nu}, f_{-\mu}^{\lambda}\right\}$ \\
\hline 188 & $i\left\{f_{+}^{\mu \nu}, f_{-\mu}^{\lambda}\right\} \gamma_{5} \gamma_{\lambda} D_{\nu}+$ H.c. & $-4 m S_{\lambda} v_{\nu}\left\{f_{+}^{\mu \nu}, f_{-\mu}^{\lambda}\right\}$ \\
\hline 189 & $i\left\langle f_{+\mu}^{\lambda} f_{-\nu \lambda}\right\rangle \gamma_{5} \gamma^{\mu} D^{\nu}+$ H.c. & $-4 m S^{\mu} v^{\nu}\left\langle f_{+\mu}^{\lambda} f_{-\nu \lambda}\right\rangle$ \\
\hline 190 & $i\left\langle f_{+\nu}^{\lambda} f_{-\mu \lambda}\right\rangle \gamma_{5} \gamma^{\mu} D^{\nu}+$ H.c. & $-4 m S^{\mu} v^{\nu}\left\langle f_{+\nu}^{\lambda} f_{-\mu \lambda}\right\rangle$ \\
\hline 191 & $i \epsilon^{\mu \nu \lambda \rho}\left[f_{+\mu \nu}, f_{-\lambda} \sigma\right] D_{\rho \sigma}+$ H.c. & $-4 i m^{2} \epsilon^{\mu \nu \lambda \rho}\left[f_{+\mu \nu}, f_{-\lambda} \sigma\right] v_{\rho} v_{\sigma}$ \\
\hline 192 & $i \epsilon^{\mu \nu \lambda \rho}\left[f_{+\mu}{ }^{\sigma}, f_{-\nu \lambda}\right] D_{\rho \sigma}+$ H.c. & $-4 i m^{2} \epsilon^{\mu \nu \lambda \rho}\left[f_{+\mu}{ }^{\sigma}, f_{-\nu \lambda}\right] v_{\rho} v_{\sigma}$ \\
\hline 193 & {$\left[D^{2}, f_{+}^{\nu \lambda}\right] \sigma_{\nu \lambda}$} & $-2 i\left[D^{2}, f_{+}^{\nu \lambda}\right]\left[S_{\nu}, S_{\lambda}\right]$ \\
\hline
\end{tabular}


TABLE V. (Continued)

\begin{tabular}{|c|c|c|}
\hline$i$ & $O_{i}^{(4)}$ & $\hat{O}_{i}^{(4)}$ \\
\hline 194 & {$\left[D_{\mu}^{\nu}, f_{+\nu \lambda}\right] \sigma^{\mu \lambda}$} & $-2 i\left[D_{\mu}^{\nu}, f_{+\nu \lambda}\right]\left[S^{\mu}, S^{\lambda}\right]$ \\
\hline 195 & {$\left[D^{\mu \nu}, f_{+}^{\lambda \rho}\right] \sigma_{\lambda \rho} D_{\mu \nu}+$ H.c. } & $8 \operatorname{im}^{2}\left[D^{\mu \nu}, f_{+}^{\lambda \rho}\right]\left[S_{\lambda}, S_{\rho}\right] v_{\mu} v_{\nu}$ \\
\hline 196 & {$\left[D^{\mu \nu}, f_{+}^{\lambda \rho}\right] \sigma_{\mu \lambda} D_{\nu \rho}+$ H.c. } & $8 \operatorname{im}^{2}\left[D^{\mu \nu}, f_{+}^{\lambda \rho}\right]\left[S_{\mu}, S_{\lambda}\right] v_{\nu} v_{\rho}$ \\
\hline 197 & $\left\{h^{\mu \nu}, h_{\mu \nu}\right\}$ & $\left\{h^{\mu \nu}, h_{\mu \nu}\right\}$ \\
\hline 198 & $\left\langle h^{\mu \nu} h_{\mu \nu}\right\rangle$ & $\left\langle h^{\mu \nu} h_{\mu \nu}\right\rangle$ \\
\hline 199 & $i\left[h^{\mu \nu}, h_{\mu}{ }^{\lambda}\right] \sigma_{\nu \lambda}$ & $2\left[h^{\mu \nu}, h_{\mu}{ }^{\lambda}\right]\left[S_{\nu}, S_{\lambda}\right]$ \\
\hline 200 & $\left\{h^{\mu \nu}, h_{\mu}{ }^{\lambda}\right\} D_{\nu \lambda}+$ H.c. & $-4 m^{2}\left\{h^{\mu \nu}, h_{\mu}^{\lambda}\right\} v_{\nu} v_{\lambda}$ \\
\hline 201 & $\left\langle h_{\mu}{ }^{\lambda} h_{\nu \lambda}\right\rangle D^{\mu \nu}+$ H.c. & $-4 m^{2} v^{\mu} v^{\nu}\left\langle h_{\mu}^{\lambda} h_{\nu \lambda}\right\rangle$ \\
\hline 202 & $i\left[h^{\mu \nu}, h^{\lambda \rho}\right] \sigma_{\mu \lambda} D_{\nu \rho}+$ H.c. & $-8 m^{2}\left[h^{\mu \nu}, h^{\lambda \rho}\right]\left[S_{\mu}, S_{\lambda}\right] v_{\nu} v_{\rho}$ \\
\hline 203 & $\left\{h^{\mu \nu}, h^{\lambda \rho}\right\} D_{\mu \nu \lambda \rho}+$ H.c. & $48 m^{4}\left\{h^{\mu \nu}, h^{\lambda \rho}\right\} v_{\mu} v_{\nu} v_{\lambda} v_{\rho}$ \\
\hline 204 & $\left\langle h_{\mu \nu} h_{\lambda \rho}\right\rangle D^{\mu \nu \lambda \rho}+$ H.c. & $48 m^{4} v^{\mu} v^{\nu} v^{\lambda} v^{\rho}\left\langle h_{\mu \nu} h_{\lambda \rho}\right\rangle$ \\
\hline 205 & $i\left[f_{-}^{\mu \nu}, h_{\mu}^{\lambda}\right] \sigma_{\nu \lambda}$ & $2\left[f_{-}^{\mu \nu}, h_{\mu}{ }^{\lambda}\right]\left[S_{\nu}, S_{\lambda}\right]$ \\
\hline 206 & $\left\{f_{-}^{\mu \nu}, h_{\mu}{ }^{\lambda}\right\} D_{\nu \lambda}+$ H.c. & $-4 m^{2} v_{\nu} v_{\lambda}\left\{f_{-}^{\mu \nu}, h_{\mu}^{\lambda}\right\}$ \\
\hline 207 & $\left\langle f_{-\mu}{ }^{\lambda} h_{\nu \lambda}\right\rangle D^{\mu \nu}+$ H.c. & $-4 m^{2} v^{\mu} v^{\nu}\left\langle f_{-\mu}^{\lambda} h_{\nu \lambda}\right\rangle$ \\
\hline 208 & $i\left[f_{-}^{\mu \nu}, h^{\lambda \rho}\right] \sigma_{\mu \nu} D_{\lambda \rho}+$ H.c. & $-8 m^{2}\left[f_{-}^{\mu \nu}, h^{\lambda \rho}\right]\left[S_{\mu}, S_{\nu}\right] v_{\lambda} v_{\rho}$ \\
\hline 209 & $i\left[f_{-}^{\mu \nu}, h^{\lambda \rho}\right] \sigma_{\mu \lambda} D_{\nu \rho}+$ H.c. & $-8 m^{2}\left[f_{-}^{\mu \nu}, h^{\lambda \rho}\right]\left[S_{\mu}, S_{\lambda}\right] v_{\nu} v_{\rho}$ \\
\hline 210 & $\left\{f_{-}^{\mu \nu}, f_{-\mu \nu}\right\}$ & $\left\{f_{-}^{\mu \nu}, f_{-\mu \nu}\right\}$ \\
\hline 211 & $\left\langle f_{-}^{\mu \nu} f_{-\mu \nu}\right\rangle$ & $\left\langle f_{-}^{\mu \nu} f_{-\mu \nu}\right\rangle$ \\
\hline 212 & $i\left[f_{-}^{\mu \nu}, f_{-\mu}{ }^{\lambda}\right] \sigma_{\nu \lambda}$ & $2\left[f_{-}^{\mu \nu}, f_{-\mu}^{\lambda}\right]\left[S_{\nu}, S_{\lambda}\right]$ \\
\hline 213 & $\left\{f_{-}^{\mu \nu}, f_{-\mu}{ }^{\lambda}\right\} D_{\nu \lambda}+$ H.c. & $-4 m^{2}\left\{f_{-}^{\mu \nu}, f_{-\mu}^{\lambda}\right\} v_{\nu} v_{\lambda}$ \\
\hline 214 & $\left\langle f_{-\mu}^{\lambda} f_{-\nu \lambda}\right\rangle D^{\mu \nu}+$ H.c. & $-4 m^{2} v^{\mu} v^{\nu}\left\langle f_{-\mu}^{\lambda} f_{-\nu \lambda}\right\rangle$ \\
\hline 215 & $i\left[f_{-}^{\mu \nu}, f_{-}^{\lambda \rho}\right] \sigma_{\mu \lambda} D_{\nu \rho}+$ H.c. & $-8 m^{2}\left[f_{-}^{\mu \nu}, f_{-}^{\lambda \rho}\right]\left[S_{\mu}, S_{\lambda}\right] v_{\nu} v_{\rho}$ \\
\hline 216 & $\left\langle\chi \chi^{\dagger}\right\rangle$ & $\left\langle\chi \chi^{\dagger}\right\rangle$ \\
\hline 217 & $\left\langle F_{R}^{\mu \nu} F_{R \mu \nu}+F_{L}^{\mu \nu} F_{L \mu \nu}\right\rangle$ & $\left\langle F_{R}^{\mu \nu} F_{R \mu \nu}+F_{L}^{\mu \nu} F_{L \mu \nu}\right\rangle$ \\
\hline 218 & $\left\langle F_{R \mu}{ }^{\lambda} F_{R \nu \lambda}+F_{L \mu}{ }^{\lambda} F_{L \nu \lambda}\right\rangle D^{\mu \nu}+$ H.c. & $-4 m^{2} v^{\mu} v^{\nu}\left\langle F_{R \mu}{ }^{\lambda} F_{R \nu \lambda}+F_{L \mu}{ }^{\lambda} F_{L \nu \lambda}\right\rangle$ \\
\hline
\end{tabular}

[1] R. Aaij et al. (LHCb Collaboration), Observation of $J / \psi p$ Resonances Consistent with Pentaquark States in $\Lambda_{b}^{0} \rightarrow$ $J / \psi K^{-} p$ Decays, Phys. Rev. Lett. 115, 072001 (2015).

[2] R. Aaij et al. (LHCb Collaboration), Observation of a Narrow Pentaquark State, $P_{c}(4312)^{+}$, and of Two-Peak Structure of the $P_{c}(4450)^{+}$, Phys. Rev. Lett. 122, 222001 (2019).

[3] P. Zyla et al. (Particle Data Group), Review of particle physics, Prog. Theor. Exp. Phys. 2020, 083 C01 (2020).

[4] M. Mattson et al. (SELEX Collaboration), First Observation of the Doubly Charmed Baryon $\Xi_{c c}^{+}$, Phys. Rev. Lett. 89, 112001 (2002).

[5] A. Ocherashvili et al. (SELEX Collaboration), Confirmation of the double charm baryon $\Xi_{c c}^{+}(3520)$ via its decay to $p D^{+} K^{-}$, Phys. Lett. B 628, 18 (2005).

[6] S. Ratti, New results on c-baryons and a search for ccbaryons in FOCUS, Nucl. Phys. B, Proc. Suppl. 115, 33 (2003).

[7] B. Aubert et al. (BABAR Collaboration), Search for doubly charmed baryons $\Xi_{c c}^{+}$and $\Xi_{c c}^{++}$in $B A B A R$, Phys. Rev. D 74, 011103 (2006).
[8] R. Chistov et al. (Belle Collaboration), Observation of New States Decaying into $\Lambda_{c}^{+} K^{-} \pi^{+}$and $\Lambda_{c}^{+} K_{s}^{0} \pi^{-}$, Phys. Rev. Lett. 97, 162001 (2006).

[9] R. Aaij et al. (LHCb Collaboration), Search for the doubly charmed baryon $\Xi_{c c}^{+}$, J. High Energy Phys. 12 (2013) 090.

[10] D. Ebert, R. Faustov, V. Galkin, and A. Martynenko, Mass spectra of doubly heavy baryons in the relativistic quark model, Phys. Rev. D 66, 014008 (2002).

[11] M. Karliner and J. L. Rosner, Baryons with two heavy quarks: Masses, production, decays, and detection, Phys. Rev. D 90, 094007 (2014).

[12] J. Korner, M. Kramer, and D. Pirjol, Heavy baryons, Prog. Part. Nucl. Phys. 33, 787 (1994).

[13] L. Liu, H.-W. Lin, K. Orginos, and A. Walker-Loud, Singly and doubly charmed $\mathbf{J}=1 / 2$ baryon spectrum from lattice QCD, Phys. Rev. D 81, 094505 (2010).

[14] Z. S. Brown, W. Detmold, S. Meinel, and K. Orginos, Charmed bottom baryon spectroscopy from lattice QCD, Phys. Rev. D 90, 094507 (2014). 
[15] S. J. Brodsky, F.-K. Guo, C. Hanhart, and U.-G. Meißner, Isospin splittings of doubly heavy baryons, Phys. Lett. B 698, 251 (2011).

[16] R. Aaij et al. (LHCb Collaboration), Observation of the Doubly Charmed Baryon $\Xi_{c c}^{++}$, Phys. Rev. Lett. 119, 112001 (2017).

[17] F.-S. Yu, H.-Y. Jiang, R.-H. Li, C.-D. Lü, W. Wang, and Z.-X. Zhao, Discovery potentials of doubly charmed baryons, Chin. Phys. C 42, 051001 (2018).

[18] W. Wang, Z.-P. Xing, and J. Xu, Weak decays of doubly heavy baryons: SU(3) analysis, Eur. Phys. J. C 77, 800 (2017).

[19] W. Wang, F.-S. Yu, and Z.-X. Zhao, Weak decays of doubly heavy baryons: The $1 / 2 \rightarrow 1 / 2$ case, Eur. Phys. J. C 77, 781 (2017).

[20] H.-S. Li, L. Meng, Z.-W. Liu, and S.-L. Zhu, Radiative decays of the doubly charmed baryons in chiral perturbation theory, Phys. Lett. B 777, 169 (2018).

[21] L.-Y. Xiao, K.-L. Wang, Q.-f. Lu, X.-H. Zhong, and S.-L. Zhu, Strong and radiative decays of the doubly charmed baryons, Phys. Rev. D 96, 094005 (2017).

[22] H.-S. Li, L. Meng, Z.-W. Liu, and S.-L. Zhu, Magnetic moments of the doubly charmed and bottom baryons, Phys. Rev. D 96, 076011 (2017).

[23] S. Weinberg, Phenomenological Lagrangians, Physica (Amsterdam) 96A, 327 (1979).

[24] J. Gasser and H. Leutwyler, Chiral perturbation theory to one loop, Ann. Phys. (N.Y.) 158, 142 (1984).

[25] J. Gasser and H. Leutwyler, Chiral perturbation theory: Expansions in the mass of the strange quark, Nucl. Phys. B250, 465 (1985).

[26] V. Bernard, N. Kaiser, and U.-G. Meißner, Chiral dynamics in nucleons and nuclei, Int. J. Mod. Phys. E 04, 193 (1995).

[27] V. Bernard, Chiral perturbation theory and baryon properties, Prog. Part. Nucl. Phys. 60, 82 (2008).

[28] E. Epelbaum, H.-W. Hammer, and U.-G. Meißner, Modern theory of nuclear forces, Rev. Mod. Phys. 81, 1773 (2009).

[29] D.-L. Yao, L.-Y. Dai, H.-Q. Zheng, and Z.-Y. Zhou, A review on partial-wave dynamics with chiral effective field theory and dispersion relation, arXiv:2009.13495.

[30] J. Gasser, M. Sainio, and A. Svarc, Nucleons with chiral loops, Nucl. Phys. B307, 779 (1988).

[31] E. E. Jenkins and A. V. Manohar, Baryon chiral perturbation theory using a heavy fermion Lagrangian, Phys. Lett. B 255, 558 (1991).

[32] V. Bernard, N. Kaiser, J. Kambor, and U. G. Meißner, Chiral structure of the nucleon, Nucl. Phys. B388, 315 (1992).

[33] P. J. Ellis and H.-B. Tang, Pion nucleon scattering in a new approach to chiral perturbation theory, Phys. Rev. C 57, 3356 (1998).

[34] T. Becher and H. Leutwyler, Baryon chiral perturbation theory in manifestly Lorentz invariant form, Eur. Phys. J. C 9, 643 (1999).

[35] T. Fuchs, J. Gegelia, G. Japaridze, and S. Scherer, Renormalization of relativistic baryon chiral perturbation theory and power counting, Phys. Rev. D 68, 056005 (2003).

[36] E. Epelbaum, J. Gegelia, U.-G. Meißner, and D.-L. Yao, Baryon chiral perturbation theory extended beyond the low-energy region, Eur. Phys. J. C 75, 499 (2015).
[37] G. Burdman and J. F. Donoghue, Union of chiral and heavy quark symmetries, Phys. Lett. B 280, 287 (1992).

[38] M. B. Wise, Chiral perturbation theory for hadrons containing a heavy quark, Phys. Rev. D 45, R2188 (1992).

[39] T.-M. Yan, H.-Y. Cheng, C.-Y. Cheung, G.-L. Lin, Y. Lin, and H.-L. Yu, Heavy quark symmetry and chiral dynamics, Phys. Rev. D 46, 1148 (1992); Erratum, Phys. Rev. D55, 5851 (1997).

[40] D. Ebert, T. Feldmann, R. Friedrich, and H. Reinhardt, Effective meson Lagrangian with chiral and heavy quark symmetries from quark flavor dynamics, Nucl. Phys. B434, 619 (1995).

[41] R. Casalbuoni, A. Deandrea, N. Di Bartolomeo, R. Gatto, F. Feruglio, and G. Nardulli, Phenomenology of heavy meson chiral Lagrangians, Phys. Rep. 281, 145 (1997).

[42] J. Hu and T. Mehen, Chiral Lagrangian with heavy quarkdiquark symmetry, Phys. Rev. D 73, 054003 (2006).

[43] P. Colangelo, F. De Fazio, F. Giannuzzi, and S. Nicotri, New meson spectroscopy with open charm and beauty, Phys. Rev. D 86, 054024 (2012).

[44] A. V. Manohar and M. B. Wise, Heavy Quark Physics (Cambridge University Press, Cambridge, 2000), Vol. 10, https://doi.org/10.1017/CBO9780511529351.

[45] L. Meng and S.-L. Zhu, Light pseudoscalar meson and doubly charmed baryon scattering lengths with heavy diquark-antiquark symmetry, Phys. Rev. D 100, 014006 (2019).

[46] S.-Z. Jiang, Y.-R. Liu, and Q.-H. Yang, Chiral Lagrangians for mesons with a single heavy quark, Phys. Rev. D 99, 074018 (2019).

[47] V. Bernard, N. Kaiser, and U.-G. Meißner, Nucleon electroweak form-factors: Analysis of their spectral functions, Nucl. Phys. A611, 429 (1996).

[48] Z.-F. Sun and M. J. Vicente Vacas, Masses of doubly charmed baryons in the extended on-mass-shell renormalization scheme, Phys. Rev. D 93, 094002 (2016).

[49] D.-L. Yao, Masses and sigma terms of doubly charmed baryons up to $\mathcal{O}\left(p^{4}\right)$ in manifestly Lorentz-invariant baryon chiral perturbation theory, Phys. Rev. D 97, 034012 (2018).

[50] A. N. Hiller Blin, Z.-F. Sun, and M. Vicente Vacas, Electromagnetic form factors of spin $1 / 2$ doubly charmed baryons, Phys. Rev. D 98, 054025 (2018).

[51] J. Goldstone, Field theories with superconductor solutions, Nuovo Cimento 19, 154 (1961).

[52] J. A. Oller, M. Verbeni, and J. Prades, Meson-baryon effective chiral lagrangians to $\mathcal{O}\left(q^{3}\right)$, J. High Energy Phys. 09 (2006) 079.

[53] S. Scherer and M.R. Schindler, A Primer for Chiral Perturbation Theory, 1st ed., Lecture Notes in Physics Vol. 830 (Springer-Verlag, Berlin, Heidelberg, 2012).

[54] A. Krause, Baryon matrix elements of the vector current in chiral perturbation theory, Helv. Phys. Acta 63, 3 (1990).

[55] N. Fettes, U.-G. Meißner, and S. Steininger, Pion-nucleon scattering in chiral perturbation theory. 1. Isospin symmetric case, Nucl. Phys. A640, 199 (1998).

[56] J. Bijnens, G. Colangelo, and G. Ecker, The mesonic chiral Lagrangian of order $p^{6}$, J. High Energy Phys. 02 (1999) 020 . 
[57] N. Fettes, U.-G. Meißner, M. Mojzis, and S. Steininger, The chiral effective pion nucleon Lagrangian of order $p^{4}$, Ann. Phys. (N.Y.) 283, 273 (2000); Erratum, Ann. Phys. (N.Y.) 288, 249 (2001).

[58] Z.-H. Guo, Prediction of exotic doubly charmed baryons within chiral effective field theory, Phys. Rev. D 96, 074004 (2017).

[59] M.-J. Yan, X.-H. Liu, S. Gonzàlez-Solís, F.-K. Guo, C. Hanhart, U.-G. Meißner, and B.-S. Zou, New spectrum of negative-parity doubly charmed baryons: Possibility of two quasistable states, Phys. Rev. D 98, 091502 (2018).
[60] D.-L. Yao, M.-L. Du, F.-K. Guo, and U.-G. Meißner, Oneloop analysis of the interactions between charmed mesons and Goldstone bosons, J. High Energy Phys. 11 (2015) 058.

[61] M. J. Savage and M. B. Wise, Spectrum of baryons with two heavy quarks, Phys. Lett. B 248, 177 (1990).

[62] Z.-F. Sun, Z.-W. Liu, X. Liu, and S.-L. Zhu, Masses and axial currents of the doubly charmed baryons, Phys. Rev. D 91, 094030 (2015).

[63] S.-Z. Jiang, Q.-S. Chen, and Y.-R. Liu, Meson-baryon effective chiral Lagrangians at order $p^{4}$, Phys. Rev. D 95, 014012 (2017). 